\title{
Investigation into the bond dissociation enthalpies (BDE) of a model lignin oligomer using density functional theory (DFT)
}

Ross W. Houston ${ }^{a}$, Thomas J. Elder ${ }^{b}$, and Nourredine H. Abdoulmouminea,c*

a. Department of Biosystems Engineering and Soil Science, University of Tennessee, 2506 E.J. Chapman Drive, Knoxville, TN 37996, USA

b. USDA-Forest Service, Southern Research Station, Auburn, AL 36849, USA

c.Center for Renewable Carbon, University of Tennessee, 2506 Jacob Drive, Knoxville, TN 37996. USA

\section{Supporting Information}

S.1. Optimized geometries of the model lignin oligomer (298 K)

\begin{tabular}{|c|c|c|c|}
\hline RRR & & & \\
\hline 0 & -8.46300 & -0.91100 & -2.92100 \\
\hline$C$ & -7.25300 & -0.70400 & -2.34500 \\
\hline$C$ & -4.76300 & -0.26500 & -1.16200 \\
\hline$C$ & -7.07500 & 0.34800 & -1.43500 \\
\hline$C$ & -6.18300 & -1.52400 & -2.65700 \\
\hline$C$ & -4.94000 & -1.30400 & -2.06700 \\
\hline$C$ & -5.84000 & 0.56700 & -0.84500 \\
\hline 0 & -8.20200 & 1.09000 & -1.21600 \\
\hline$C$ & -8.11600 & 2.15000 & -0.28500 \\
\hline$C$ & -3.42800 & -0.08500 & -0.48300 \\
\hline$C$ & -3.14200 & -1.13700 & 0.60300 \\
\hline O & -2.69700 & -2.35100 & -0.03300 \\
\hline$C$ & -4.33600 & -1.48800 & 1.46600 \\
\hline $\mathrm{O}$ & -3.95400 & -2.24700 & 2.59100 \\
\hline O & -3.38000 & 1.21900 & 0.10600 \\
\hline$C$ & -1.34500 & -2.41100 & -0.24000 \\
\hline$C$ & 1.42700 & -2.57900 & -0.61400 \\
\hline$C$ & -0.49800 & -2.69300 & 0.85000 \\
\hline$C$ & -0.79900 & -2.22400 & -1.49600 \\
\hline$C$ & 0.58100 & -2.29600 & -1.68300 \\
\hline
\end{tabular}




\begin{tabular}{|c|c|c|c|}
\hline 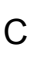 & 0.87100 & -2.78300 & 0.65900 \\
\hline ; & 2.87800 & -2.66100 & -0.85800 \\
\hline C & 3.79800 & -3.15600 & -0.02700 \\
\hline C & 5.26300 & -3.24500 & -0.34300 \\
\hline $\mathrm{O}$ & 5.61600 & -2.69800 & -1.59900 \\
\hline $\mathrm{O}$ & -1.12200 & -2.85400 & 2.04300 \\
\hline C & -0.31600 & -2.96500 & 3.20500 \\
\hline C & -2.12000 & 1.63600 & 0.45700 \\
\hline C & 0.44600 & 2.43600 & 1.20900 \\
\hline C & -1.17300 & 1.95100 & -0.51000 \\
\hline C & -1.79200 & 1.74300 & 1.81300 \\
\hline C & -0.51600 & 2.15600 & 2.17600 \\
\hline C & 0.10700 & 2.34300 & -0.13800 \\
\hline $\mathrm{O}$ & -2.67300 & 1.36800 & 2.78500 \\
\hline C & -3.82700 & 2.19400 & 2.91600 \\
\hline$C$ & 1.85800 & 2.76400 & 1.63900 \\
\hline $\mathrm{O}$ & 2.48800 & 3.57000 & 0.62300 \\
\hline$C$ & 2.77700 & 1.51000 & 1.84700 \\
\hline$C$ & 3.75500 & 1.64900 & 0.70300 \\
\hline C & 5.26800 & 2.42900 & -1.46800 \\
\hline$C$ & 3.48800 & 2.84700 & 0.05200 \\
\hline C & 4.81200 & 0.85000 & 0.30200 \\
\hline C & 5.59400 & 1.25100 & -0.79100 \\
\hline C & 4.22300 & 3.25400 & -1.05900 \\
\hline $\mathrm{O}$ & 4.01800 & 4.42600 & -1.72000 \\
\hline C & 2.03200 & 0.17000 & 1.86800 \\
\hline $\mathrm{O}$ & 2.92500 & -0.90200 & 2.10800 \\
\hline C & 2.67500 & 4.70700 & -2.10200 \\
\hline C & 6.75800 & 0.47000 & -1.24800 \\
\hline C & 7.46100 & -0.40200 & -0.51900 \\
\hline C & 8.62300 & -1.19100 & -1.05400 \\
\hline O & 8.42200 & -2.58500 & -0.91900 \\
\hline
\end{tabular}




\begin{tabular}{|c|c|c|c|}
\hline 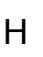 & -9.07400 & -0.25000 & -2.57700 \\
\hline 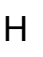 & -6.33800 & -2.33000 & -3.36300 \\
\hline 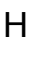 & -4.11100 & -1.96200 & -2.30000 \\
\hline I & -5.69100 & 1.38200 & -0.15000 \\
\hline 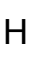 & -7.38500 & 2.89600 & -0.61100 \\
\hline 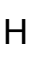 & -7.84000 & 1.77500 & 0.70500 \\
\hline 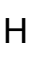 & -9.10400 & 2.60000 & -0.24300 \\
\hline 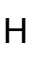 & -2.63000 & -0.16500 & -1.22900 \\
\hline 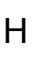 & -2.34300 & -0.76500 & 1.25200 \\
\hline 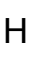 & -5.07400 & -2.02300 & 0.85700 \\
\hline $\mathrm{H}$ & -4.79000 & -0.56100 & 1.82800 \\
\hline $\mathrm{H}$ & -3.25900 & -2.85300 & 2.30700 \\
\hline $\mathrm{H}$ & -1.46500 & -2.02300 & -2.32700 \\
\hline $\mathrm{H}$ & 1.00000 & -2.14100 & -2.67100 \\
\hline $\mathrm{H}$ & 1.51600 & -2.98100 & 1.50400 \\
\hline $\mathrm{H}$ & 3.20700 & -2.31300 & -1.83400 \\
\hline $\mathrm{H}$ & 3.51600 & -3.56300 & 0.94300 \\
\hline $\mathrm{H}$ & 5.56400 & -4.29600 & -0.37200 \\
\hline $\mathrm{H}$ & 5.85100 & -2.77800 & 0.46100 \\
\hline $\mathrm{H}$ & 5.55800 & -1.73600 & -1.54100 \\
\hline $\mathrm{H}$ & 0.23900 & -3.90700 & 3.20400 \\
\hline $\mathrm{H}$ & 0.38400 & -2.12800 & 3.27300 \\
\hline $\mathrm{H}$ & -1.00400 & -2.94300 & 4.04700 \\
\hline $\mathrm{H}$ & -1.45000 & 1.87600 & -1.55500 \\
\hline $\mathrm{H}$ & -0.28600 & 2.22400 & 3.23500 \\
\hline $\mathrm{H}$ & 0.84500 & 2.57500 & -0.89700 \\
\hline $\mathrm{H}$ & -3.53300 & 3.21100 & 3.19100 \\
\hline 11 & -4.42300 & 1.75600 & 3.71400 \\
\hline 11 & -4.40200 & 2.21100 & 1.98800 \\
\hline 11 & 1.82700 & 3.37000 & 2.54600 \\
\hline $\mathrm{H}$ & 3.31000 & 1.59900 & 2.79800 \\
\hline $\mathrm{H}$ & 5.85100 & 2.75100 & -2.32400 \\
\hline
\end{tabular}




$\begin{array}{llll}\mathrm{H} & 5.05000 & -0.05800 & 0.84500 \\ \mathrm{H} & 1.48400 & 0.02000 & 0.93100 \\ \mathrm{H} & 1.30200 & 0.16900 & 2.68100 \\ \mathrm{H} & 3.22100 & -1.24000 & 1.25600 \\ \mathrm{H} & 2.04700 & 4.90900 & -1.23300 \\ \mathrm{H} & 2.26300 & 3.86800 & -2.67200 \\ \mathrm{H} & 2.72200 & 5.58700 & -2.73900 \\ \mathrm{H} & 7.07400 & 0.65400 & -2.27400 \\ \mathrm{H} & 7.20600 & -0.58600 & 0.52300 \\ \mathrm{H} & 9.52500 & -0.96300 & -0.48100 \\ \mathrm{H} & 8.81000 & -0.91200 & -2.09900 \\ \mathrm{H} & 7.61000 & -2.81100 & -1.39200\end{array}$

RRRS

$\begin{array}{llll}\text { O } & -9.14100 & -0.10600 & -2.02300 \\ C & -7.85700 & -0.08400 & -1.58600 \\ C & -5.21900 & -0.02400 & -0.68200 \\ C & -7.52500 & 0.60600 & -0.41000 \\ C & -6.86600 & -0.73500 & -2.29900 \\ C & -5.54800 & -0.70200 & -1.85000 \\ C & -6.21500 & 0.63500 & 0.04300 \\ \text { O } & -8.58900 & 1.21200 & 0.19700 \\ C & -8.34400 & 1.89900 & 1.40900 \\ C & -3.79500 & -0.05600 & -0.18800 \\ C & -3.38400 & -1.38700 & 0.47000 \\ \text { O } & -2.84600 & -2.26400 & -0.53700 \\ C & -4.49500 & -2.14200 & 1.16800 \\ \text { O } & -3.97600 & -3.17500 & 1.97700 \\ \text { O } & -3.60500 & 1.01800 & 0.74900 \\ \text { C } & -1.50900 & -2.07200 & -0.78400 \\ \text { C } & 1.23000 & -1.78000 & -1.28000 \\ \text { C } & -0.56700 & -2.52800 & 0.15900 \\ \text { C } & -1.07200 & -1.45700 & -1.94300\end{array}$




\begin{tabular}{|c|c|c|c|}
\hline 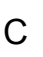 & 0.29200 & -1.30100 & -2.19000 \\
\hline ; & 0.78800 & -2.37500 & -0.08700 \\
\hline C & 2.67500 & -1.68000 & -1.56300 \\
\hline C & 3.58200 & -2.55300 & -1.12400 \\
\hline C & 5.05000 & -2.48200 & -1.39600 \\
\hline $\mathrm{O}$ & 5.72300 & -2.66000 & -0.15200 \\
\hline O & -1.08600 & -3.10100 & 1.27600 \\
\hline C & -0.18400 & -3.43600 & 2.31800 \\
\hline C & -2.28100 & 1.28400 & 1.00300 \\
\hline C & 0.43300 & 1.65800 & 1.53500 \\
\hline C & -1.78300 & 1.08200 & 2.28300 \\
\hline C & -1.42400 & 1.74000 & -0.01300 \\
\hline C & -0.07100 & 1.89300 & 0.25800 \\
\hline C & -0.43300 & 1.27900 & 2.55600 \\
\hline $\mathrm{O}$ & -1.85200 & 2.00000 & -1.28100 \\
\hline C & -2.99800 & 2.84200 & -1.41900 \\
\hline C & 1.91400 & 1.74800 & 1.77300 \\
\hline $\mathrm{O}$ & 2.46200 & 2.86100 & 1.03400 \\
\hline C & 2.68100 & 0.48400 & 1.30300 \\
\hline C & 3.88600 & 1.09900 & 0.64200 \\
\hline C & 5.71800 & 2.69400 & -0.66600 \\
\hline C & 3.64500 & 2.45900 & 0.48500 \\
\hline C & 5.05800 & 0.53300 & 0.18700 \\
\hline C & 6.00100 & 1.34300 & -0.46100 \\
\hline C & 4.54400 & 3.28200 & -0.19100 \\
\hline $\mathrm{O}$ & 4.37400 & 4.61800 & -0.38400 \\
\hline C & 2.95700 & -0.48700 & 2.45100 \\
\hline $\mathrm{O}$ & 3.28100 & -1.79500 & 2.02300 \\
\hline C & 3.06100 & 5.05800 & -0.71700 \\
\hline C & 7.24300 & 0.72700 & -0.96000 \\
\hline C & 7.83800 & -0.29700 & -0.34800 \\
\hline C & 8.97700 & -1.08400 & -0.90100 \\
\hline
\end{tabular}




\begin{tabular}{|c|c|c|c|}
\hline 0 & 8.49500 & -2.42800 & -1.05400 \\
\hline 4 & -9.67800 & 0.39000 & -1.39400 \\
\hline 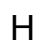 & -7.14200 & -1.26000 & -3.20600 \\
\hline $\mathrm{H}$ & -4.77500 & -1.22200 & -2.40500 \\
\hline 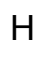 & -5.94400 & 1.16900 & 0.94400 \\
\hline $\mathrm{H}$ & -7.94800 & 1.21800 & 2.16800 \\
\hline $\mathrm{H}$ & -9.30300 & 2.29200 & 1.73500 \\
\hline $\mathrm{H}$ & -7.64200 & 2.72300 & 1.25400 \\
\hline $\mathrm{H}$ & -3.12300 & 0.09600 & -1.03800 \\
\hline $\mathrm{H}$ & -2.59900 & -1.17900 & 1.20200 \\
\hline $\mathrm{H}$ & -5.19200 & -2.53300 & 0.41800 \\
\hline $\mathrm{H}$ & -5.04300 & -1.45400 & 1.81800 \\
\hline $\mathrm{H}$ & -3.22000 & -3.55400 & 1.51100 \\
\hline $\mathrm{H}$ & -1.81000 & -1.10600 & -2.65400 \\
\hline $\mathrm{H}$ & 0.62300 & -0.82400 & -3.10500 \\
\hline $\mathrm{H}$ & 1.52200 & -2.68200 & 0.64800 \\
\hline $\mathrm{H}$ & 2.99500 & -0.84600 & -2.18700 \\
\hline $\mathrm{H}$ & 3.27100 & -3.40400 & -0.52100 \\
\hline $\mathrm{H}$ & 5.34200 & -3.27900 & -2.09000 \\
\hline $\mathrm{H}$ & 5.31500 & -1.51800 & -1.85000 \\
\hline $\mathrm{H}$ & 6.67100 & -2.72400 & -0.33600 \\
\hline $\mathrm{H}$ & 0.39600 & -2.56100 & 2.62500 \\
\hline $\mathrm{H}$ & -0.80000 & -3.78300 & 3.14400 \\
\hline $\mathrm{H}$ & 0.50100 & -4.22800 & 2.00400 \\
\hline$H$ & -2.47000 & 0.74400 & 3.05000 \\
\hline$H$ & 0.57900 & 2.22000 & -0.54500 \\
\hline$H$ & -0.05600 & 1.12300 & 3.56100 \\
\hline$H$ & -3.01500 & 3.59500 & -0.62700 \\
\hline$H$ & -3.92500 & 2.26600 & -1.39100 \\
\hline$H$ & -2.89800 & 3.33000 & -2.38700 \\
\hline $\mathrm{H}$ & 2.11700 & 1.93400 & 2.83600 \\
\hline $\mathrm{H}$ & 2.08000 & -0.04800 & 0.55800 \\
\hline
\end{tabular}




$\begin{array}{llll}\mathrm{H} & 6.41900 & 3.33600 & -1.18800 \\ \mathrm{H} & 5.24700 & -0.52900 & 0.28600 \\ \mathrm{H} & 2.04500 & -0.59500 & 3.04600 \\ \mathrm{H} & 3.73800 & -0.07600 & 3.10300 \\ \mathrm{H} & 4.11100 & -1.80600 & 1.52900 \\ \mathrm{H} & 2.40600 & 5.05500 & 0.15400 \\ \mathrm{H} & 2.63200 & 4.42100 & -1.49700 \\ \mathrm{H} & 3.17400 & 6.07200 & -1.09800 \\ \mathrm{H} & 7.65700 & 1.11500 & -1.88900 \\ \mathrm{H} & 7.44700 & -0.65500 & 0.60200 \\ \mathrm{H} & 9.82900 & -1.07800 & -0.21300 \\ \mathrm{H} & 9.29700 & -0.68100 & -1.86700 \\ \mathrm{H} & 9.23300 & -3.02400 & -1.20600\end{array}$

RRSR

$\begin{array}{llll}\text { O } & 9.23800 & -1.59400 & -1.10600 \\ \text { C } & 7.94200 & -1.27800 & -0.85900 \\ \text { C } & 5.27900 & -0.63200 & -0.33500 \\ \text { C } & 7.60500 & -0.51500 & 0.26800 \\ \text { C } & 6.94400 & -1.71500 & -1.71200 \\ \text { C } & 5.61400 & -1.39500 & -1.44700 \\ \text { C } & 6.28300 & -0.19000 & 0.53000 \\ \text { O } & 8.67700 & -0.15500 & 1.03600 \\ \text { C } & 8.42200 & 0.62300 & 2.18800 \\ \text { C } & 3.83700 & -0.25400 & -0.11300 \\ \text { C } & 3.31100 & 0.84000 & -1.06900 \\ \text { O } & 2.59700 & 0.23200 & -2.16400 \\ \text { C } & 4.34800 & 1.74600 & -1.69700 \\ \text { O } & 3.72900 & 2.86100 & -2.30400 \\ \text { O } & 3.67900 & 0.18500 & 1.24800 \\ \text { C } & 1.31600 & -0.16300 & -1.88300 \\ \text { C } & -1.34200 & -0.98000 & -1.53500 \\ \text { C } & 0.30700 & 0.79700 & -1.66800\end{array}$




\begin{tabular}{|c|c|c|c|}
\hline C & 0.98600 & -1.50900 & -1.90000 \\
\hline C & -0.33300 & -1.92200 & -1.73200 \\
\hline C & -1.00900 & 0.38100 & -1.49800 \\
\hline C & -2.75900 & -1.36300 & -1.40000 \\
\hline C & -3.21000 & -2.54000 & -0.96400 \\
\hline C & -4.66500 & -2.87500 & -0.88800 \\
\hline ) & -4.99500 & -3.18500 & 0.47400 \\
\hline 0 & 0.70800 & 2.09000 & -1.69800 \\
\hline C & -0.25500 & 3.08400 & -1.37900 \\
\hline 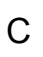 & 2.36600 & 0.34300 & 1.60700 \\
\hline C & -0.34900 & 0.73500 & 2.10700 \\
\hline 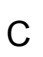 & 1.87200 & 1.61600 & 1.84600 \\
\hline C & 1.50700 & -0.76500 & 1.69700 \\
\hline C & 0.15100 & -0.55700 & 1.93900 \\
\hline 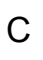 & 0.52100 & 1.81900 & 2.10200 \\
\hline 0 & 2.06500 & -1.98300 & 1.50500 \\
\hline$C$ & 1.21100 & -3.11200 & 1.56100 \\
\hline C & -1.83100 & 0.98100 & 2.24300 \\
\hline 0 & -2.19100 & 2.08000 & 1.35500 \\
\hline C & -2.73700 & -0.19500 & 1.84600 \\
\hline C & -3.86300 & 0.51700 & 1.14500 \\
\hline C & -5.37400 & 2.14800 & -0.47400 \\
\hline C & -3.41900 & 1.79900 & 0.83800 \\
\hline C & -5.07400 & 0.05100 & 0.68500 \\
\hline C & -5.85000 & 0.87400 & -0.14700 \\
\hline C & -4.16000 & 2.63800 & 0.01200 \\
\hline O & -3.74000 & 3.86900 & -0.39800 \\
\hline C & -3.09000 & -1.15800 & 2.98700 \\
\hline O & -3.08000 & -2.50700 & 2.57200 \\
\hline $\mathrm{C}$ & -3.23500 & 4.73400 & 0.61700 \\
\hline $\mathrm{C}$ & -7.12100 & 0.40800 & -0.7230 \\
\hline $\mathrm{C}$ & -7.71500 & -0.76200 & -0.47300 \\
\hline
\end{tabular}




\begin{tabular}{|c|c|c|c|}
\hline C & -8.97100 & -1.22200 & -1.14500 \\
\hline 0 & -8.75200 & -2.42200 & -1.88000 \\
\hline & 9.77700 & -1.20400 & -0.40700 \\
\hline & 7.22200 & -2.30800 & -2.57400 \\
\hline | & 4.83400 & -1.73300 & -2.12000 \\
\hline | & 6.00700 & 0.38900 & 1.40100 \\
\hline y & 7.77000 & 0.08600 & 2.88400 \\
\hline I & 7.96100 & 1.57700 & 1.91800 \\
\hline y & 9.38700 & 0.80200 & 2.65500 \\
\hline † & 3.22000 & -1.14200 & -0.26500 \\
\hline - & 2.61300 & 1.47400 & -0.52200 \\
\hline$H$ & 4.94700 & 1.17900 & -2.41900 \\
\hline $\mathrm{H}$ & 5.01800 & 2.12200 & -0.92000 \\
\hline $\mathrm{H}$ & 2.93600 & 2.54100 & -2.74900 \\
\hline $\mathrm{H}$ & 1.77800 & -2.22400 & -2.08800 \\
\hline $\mathrm{H}$ & -0.57800 & -2.97500 & -1.80500 \\
\hline $\mathrm{H}$ & -1.79800 & 1.11100 & -1.35100 \\
\hline $\mathrm{H}$ & -3.48400 & -0.59100 & -1.65600 \\
\hline $\mathrm{H}$ & -2.52300 & -3.31300 & -0.62800 \\
\hline $\mathrm{H}$ & -4.88600 & -3.74300 & -1.51700 \\
\hline $\mathrm{H}$ & -5.26800 & -2.02800 & -1.23500 \\
\hline $\mathrm{H}$ & -5.75000 & -3.77800 & 0.49900 \\
\hline $\mathrm{H}$ & -0.69700 & 2.88900 & -0.39800 \\
\hline $\mathrm{H}$ & 0.28800 & 4.02700 & -1.36700 \\
\hline $\mathrm{H}$ & -1.04300 & 3.12400 & -2.13600 \\
\hline $\mathrm{H}$ & 2.56300 & 2.44900 & 1.77800 \\
\hline 1 & -0.51600 & -1.40800 & 1.97300 \\
\hline $\mathrm{H}$ & 0.13800 & 2.82200 & 2.24300 \\
\hline 1 & 0.74600 & -3.20500 & 2.54600 \\
\hline $\mathrm{H}$ & 1.84700 & -3.97500 & 1.37500 \\
\hline $\mathrm{H}$ & 0.43400 & -3.05000 & 0.79300 \\
\hline$H$ & -2.07800 & 1.31700 & 3.25900 \\
\hline
\end{tabular}




\begin{tabular}{|c|c|c|c|}
\hline $\mathrm{H}$ & -2.22200 & -0.78900 & 1.08500 \\
\hline $\mathrm{H}$ & -5.94800 & 2.79900 & -1.12300 \\
\hline $\mathrm{H}$ & -5.39000 & -0.95600 & 0.93100 \\
\hline $\mathrm{H}$ & -4.05400 & -0.87900 & 3.43000 \\
\hline $\mathrm{H}$ & -2.33200 & -1.08500 & 3.77300 \\
\hline $\mathrm{H}$ & -3.76900 & -2.66400 & 1.91000 \\
\hline $\mathrm{H}$ & -2.25400 & 4.40900 & 0.96400 \\
\hline $\mathrm{H}$ & -3.92700 & 4.77100 & 1.46300 \\
\hline $\mathrm{H}$ & -3.16400 & 5.72000 & 0.16200 \\
\hline $\mathrm{H}$ & -7.59700 & 1.09100 & -1.42500 \\
\hline $\mathrm{H}$ & -7.27500 & -1.47100 & 0.22400 \\
\hline $\mathrm{H}$ & -9.37100 & -0.43100 & -1.79000 \\
\hline $\mathrm{H}$ & -9.73700 & -1.47500 & -0.40800 \\
\hline $\mathrm{H}$ & -8.09100 & -2.23600 & -2.55200 \\
\hline \multicolumn{4}{|l|}{ RRSS } \\
\hline $\mathrm{O}$ & -8.70100 & -0.42900 & -0.29500 \\
\hline$C$ & -7.39100 & -0.17000 & -0.05500 \\
\hline$C$ & -4.69500 & 0.35500 & 0.42400 \\
\hline$C$ & -6.41200 & -1.11500 & -0.40300 \\
\hline $\mathrm{C}$ & -7.01100 & 1.02400 & 0.53000 \\
\hline C & -5.66200 & 1.28500 & 0.77500 \\
\hline C & -5.07300 & -0.85500 & -0.17100 \\
\hline $\mathrm{O}$ & -6.90900 & -2.25400 & -0.97100 \\
\hline$C$ & -5.98100 & -3.27300 & -1.29200 \\
\hline$C$ & -3.23100 & 0.56500 & 0.70700 \\
\hline$C$ & -2.71800 & -0.20100 & 1.93800 \\
\hline $\mathrm{O}$ & -2.52500 & -1.58200 & 1.59800 \\
\hline$C$ & -3.64700 & -0.17100 & 3.13400 \\
\hline $\mathrm{O}$ & -3.05200 & -0.81200 & 4.24400 \\
\hline $\mathrm{O}$ & -2.95200 & 1.96000 & 0.91600 \\
\hline$C$ & -1.25400 & -1.91900 & 1.20400 \\
\hline C & 1.30200 & -2.75500 & 0.41100 \\
\hline
\end{tabular}




\begin{tabular}{|c|c|c|c|}
\hline C & -0.98400 & -2.16000 & -0.15400 \\
\hline ; & -0.24300 & -2.06200 & 2.13900 \\
\hline C & 1.02700 & -2.47400 & 1.74900 \\
\hline C & 0.28200 & -2.58500 & -0.53900 \\
\hline C & 2.64800 & -3.24600 & 0.06100 \\
\hline C & 3.12800 & -3.50400 & -1.15700 \\
\hline C & 4.50900 & -4.03900 & -1.40200 \\
\hline O & 5.10400 & -4.46200 & -0.19900 \\
\hline O & -2.01800 & -1.96200 & -1.01500 \\
\hline C & -1.71700 & -1.91800 & -2.39900 \\
\hline C & -1.79500 & 2.35100 & 0.29400 \\
\hline C & 0.60400 & 2.94000 & -1.00200 \\
\hline C & -0.66100 & 2.65300 & 1.03200 \\
\hline$C$ & -1.75100 & 2.40500 & -1.10800 \\
\hline$C$ & -0.55700 & 2.71100 & -1.74100 \\
\hline$c$ & 0.53900 & 2.94300 & 0.38700 \\
\hline $\mathrm{O}$ & -2.84500 & 2.06700 & -1.85100 \\
\hline$C$ & -3.92600 & 3.00000 & -1.78800 \\
\hline$C$ & 1.92200 & 3.03300 & -1.73500 \\
\hline $\mathrm{O}$ & 2.88300 & 3.75300 & -0.93900 \\
\hline C & 2.55800 & 1.62900 & -2.01000 \\
\hline$C$ & 3.62500 & 1.57500 & -0.94200 \\
\hline C & 5.54000 & 2.10400 & 0.97200 \\
\hline C & 3.74100 & 2.84700 & -0.39300 \\
\hline C & 4.47700 & 0.55900 & -0.55100 \\
\hline C & 5.44400 & 0.81900 & 0.43200 \\
\hline C & 4.69600 & 3.14000 & 0.57600 \\
\hline $\mathrm{O}$ & 4.88300 & 4.38100 & 1.10500 \\
\hline C & 1.57000 & 0.45700 & -1.99400 \\
\hline $\mathrm{O}$ & 2.21400 & -0.75800 & -2.33300 \\
\hline C & 3.72100 & 5.00500 & 1.64500 \\
\hline C & 6.36700 & -0.22800 & 0.89600 \\
\hline
\end{tabular}




\begin{tabular}{|c|c|c|c|}
\hline C & 6.17700 & -1.54200 & 0.76400 \\
\hline C & 7.16700 & -2.57000 & 1.21200 \\
\hline & 7.65000 & -3.34700 & 0.10700 \\
\hline & -8.76300 & -1.30700 & -0.69000 \\
\hline & -7.78100 & 1.73800 & 0.79300 \\
\hline & -5.36500 & 2.21900 & 1.23900 \\
\hline | & -4.30200 & -1.57200 & -0.42700 \\
\hline | & -5.26500 & -2.92400 & -2.04200 \\
\hline - & -6.56300 & -4.09800 & -1.69600 \\
\hline † & -5.44000 & -3.60000 & -0.40000 \\
\hline - & -2.65800 & 0.20800 & -0.15200 \\
\hline-1 & -1.75100 & 0.22800 & 2.22700 \\
\hline$H$ & -4.60100 & -0.64000 & 2.87000 \\
\hline$H$ & -3.84000 & 0.86600 & 3.41600 \\
\hline 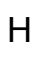 & -2.94100 & -1.73700 & 4.00400 \\
\hline 1 & -0.46900 & -1.85000 & 3.17900 \\
\hline$H$ & 1.80700 & -2.59500 & 2.49100 \\
\hline$H$ & 0.48300 & -2.79200 & -1.58100 \\
\hline $\mathrm{H}$ & 3.30400 & -3.44200 & 0.90500 \\
\hline 1 & 2.53100 & -3.34200 & -2.05200 \\
\hline 11 & 5.11400 & -3.25600 & -1.88700 \\
\hline$\pi$ & 4.44200 & -4.87100 & -2.11700 \\
\hline $\mathrm{H}$ & 6.06000 & -4.32900 & -0.26000 \\
\hline $\mathrm{H}$ & -2.61900 & -1.55800 & -2.89000 \\
\hline $\mathrm{H}$ & -0.89100 & -1.22700 & -2.59500 \\
\hline 1 & -1.46000 & -2.91100 & -2.77900 \\
\hline 1 & -0.72400 & 2.62100 & 2.11400 \\
\hline T & -0.54400 & 2.72600 & -2.82700 \\
\hline 1 & 1.43100 & 3.13700 & 0.97000 \\
\hline $\mathrm{H}$ & -3.65400 & 3.91700 & -2.31900 \\
\hline $\mathrm{H}$ & -4.18300 & 3.22600 & -0.75200 \\
\hline$H$ & -4.77400 & 2.52200 & -2.27400 \\
\hline
\end{tabular}




$\begin{array}{llll}\mathrm{H} & 1.79300 & 3.59500 & -2.66000 \\ \mathrm{H} & 3.03700 & 1.63300 & -2.99500 \\ \mathrm{H} & 6.28700 & 2.33100 & 1.72400 \\ \mathrm{H} & 4.44200 & -0.41000 & -1.03400 \\ \mathrm{H} & 0.79600 & 0.62400 & -2.74700 \\ \mathrm{H} & 1.07800 & 0.37600 & -1.01800 \\ \mathrm{H} & 2.50000 & -1.19100 & -1.52100 \\ \mathrm{H} & 3.26400 & 4.35900 & 2.40100 \\ \mathrm{H} & 2.99400 & 5.23600 & 0.86500 \\ \mathrm{H} & 4.06400 & 5.92400 & 2.11600 \\ \mathrm{H} & 7.26600 & 0.12300 & 1.40100 \\ \mathrm{H} & 5.27000 & -1.93300 & 0.30800 \\ \mathrm{H} & 6.69900 & -3.29700 & 1.87900 \\ \mathrm{H} & 8.00400 & -2.10300 & 1.73900 \\ \mathrm{H} & 8.04700 & -2.74600 & -0.53000\end{array}$

RSRR

$\begin{array}{llll}\text { O } & -8.01700 & -1.73900 & -3.04200 \\ C & -6.85600 & -1.36300 & -2.45200 \\ C & -4.48000 & -0.58100 & -1.22200 \\ C & -6.86900 & -0.83600 & -1.14900 \\ C & -5.65700 & -1.49200 & -3.12600 \\ \text { C } & -4.46800 & -1.09900 & -2.50900 \\ \text { C } & -5.69200 & -0.44600 & -0.53600 \\ \text { O } & -8.11200 & -0.76700 & -0.58900 \\ \text { C } & -8.19100 & -0.30800 & 0.75000 \\ C & -3.18700 & -0.23000 & -0.53600 \\ \text { C } & -2.62100 & -1.42500 & 0.25600 \\ \text { O } & -1.47800 & -0.97100 & 1.00800 \\ \text { C } & -3.58500 & -2.09300 & 1.22700 \\ \text { O } & -4.19000 & -1.21300 & 2.14800 \\ \text { O } & -3.40500 & 0.89200 & 0.33200 \\ \text { C } & -0.28400 & -1.59300 & 0.77200\end{array}$




\begin{tabular}{|c|c|c|c|}
\hline C & 2.22500 & -2.77800 & 0.35500 \\
\hline C & 0.47400 & -1.25700 & -0.36700 \\
\hline C & 0.22900 & -2.49500 & 1.69200 \\
\hline C & 1.47300 & -3.09300 & 1.48800 \\
\hline C & 1.71700 & -1.84700 & -0.56300 \\
\hline C & 3.52100 & -3.42700 & 0.07800 \\
\hline C & 4.42700 & -3.77800 & 0.99000 \\
\hline C & 5.70900 & -4.49200 & 0.66000 \\
\hline $\mathrm{O}$ & 6.84800 & -3.75500 & 1.05400 \\
\hline O & -0.08700 & -0.35400 & -1.20500 \\
\hline C & 0.75800 & 0.27900 & -2.15500 \\
\hline$C$ & -2.30400 & 1.65500 & 0.60400 \\
\hline C & 0.07300 & 2.95300 & 1.26000 \\
\hline$C$ & -1.53500 & 2.23800 & -0.38200 \\
\hline$C$ & -1.94600 & 1.82100 & 1.95200 \\
\hline C & -0.77800 & 2.49400 & 2.27300 \\
\hline$C$ & -0.33100 & 2.86300 & -0.06200 \\
\hline O & -2.77300 & 1.22900 & 2.85500 \\
\hline C & -2.33100 & 1.15000 & 4.19800 \\
\hline C & 1.47900 & 3.37400 & 1.63100 \\
\hline $\mathrm{O}$ & 2.08400 & 4.10100 & 0.54000 \\
\hline C & 2.42300 & 2.14900 & 1.86800 \\
\hline C & 3.06800 & 2.02500 & 0.50900 \\
\hline$\checkmark$ & 4.07800 & 2.38200 & -2.02500 \\
\hline 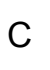 & 2.82800 & 3.21200 & -0.17800 \\
\hline 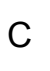 & 3.81800 & 1.01200 & -0.05100 \\
\hline 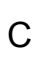 & 4.33100 & 1.18600 & -1.34700 \\
\hline$\checkmark$ & 3.33200 & 3.41800 & -1.46000 \\
\hline 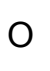 & 3.19200 & 4.57800 & -2.16200 \\
\hline$\checkmark$ & 1.75200 & 0.86500 & 2.34500 \\
\hline 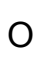 & 2.76500 & -0.10900 & 2.52700 \\
\hline C & 1.86200 & 5.06200 & -2.31200 \\
\hline
\end{tabular}




\begin{tabular}{|c|c|c|c|}
\hline $\mathrm{C}$ & 5.12700 & 0.14100 & -2.00700 \\
\hline 0 & 5.46000 & -1.03900 & -1.47500 \\
\hline 0 & 6.29500 & -2.06300 & -2.17400 \\
\hline 0 & 7.45600 & -2.39500 & -1.40800 \\
\hline 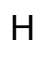 & -8.73400 & -1.57700 & -2.41600 \\
\hline $\mathrm{H}$ & -5.66800 & -1.89400 & -4.13200 \\
\hline $\mathrm{H}$ & -3.52900 & -1.19800 & -3.04400 \\
\hline - & -5.68100 & -0.05600 & 0.47200 \\
\hline † & -9.24000 & -0.36900 & 1.02900 \\
\hline | & -7.84800 & 0.72700 & 0.82500 \\
\hline $\mathrm{H}$ & -7.59100 & -0.93900 & 1.41200 \\
\hline $\mathrm{H}$ & -2.43600 & 0.03400 & -1.28200 \\
\hline $\mathrm{H}$ & -2.30100 & -2.17700 & -0.47500 \\
\hline$H$ & -3.02100 & -2.87400 & 1.75500 \\
\hline$H$ & -4.38100 & -2.58100 & 0.65900 \\
\hline $\mathrm{H}$ & -3.64800 & -0.41800 & 2.25700 \\
\hline $\mathrm{H}$ & -0.37000 & -2.72700 & 2.56500 \\
\hline $\mathrm{H}$ & 1.83900 & -3.83400 & 2.18800 \\
\hline - & 2.32100 & -1.57900 & -1.42300 \\
\hline $\mathrm{H}$ & 3.73200 & -3.62300 & -0.97300 \\
\hline $\mathrm{H}$ & 4.27300 & -3.53500 & 2.04000 \\
\hline $\mathrm{H}$ & 5.73700 & -4.72700 & -0.41200 \\
\hline $\mathrm{H}$ & 5.74000 & -5.44100 & 1.20300 \\
\hline $\mathrm{H}$ & 7.11700 & -3.20800 & 0.30400 \\
\hline $\mathrm{H}$ & 1.10400 & -0.43000 & -2.91300 \\
\hline $\mathrm{H}$ & 0.14600 & 1.04300 & -2.63200 \\
\hline $\mathrm{H}$ & 1.61300 & 0.74800 & -1.66100 \\
\hline $\mathrm{H}$ & -1.84700 & 2.14300 & -1.41600 \\
\hline $\mathrm{H}$ & -0.47900 & 2.60100 & 3.30900 \\
\hline$H$ & 0.29900 & 3.25000 & -0.85100 \\
\hline $\mathrm{H}$ & -2.26400 & 2.14300 & 4.64900 \\
\hline $\mathrm{H}$ & -3.07900 & 0.56100 & 4.72400 \\
\hline
\end{tabular}




$\begin{array}{rrrr}\mathrm{H} & -1.36000 & 0.64800 & 4.25400 \\ \mathrm{H} & 1.46900 & 4.04600 & 2.49000 \\ \mathrm{H} & 3.18900 & 2.42300 & 2.60200 \\ \mathrm{H} & 4.47000 & 2.54300 & -3.02300 \\ \mathrm{H} & 3.99700 & 0.11000 & 0.51700 \\ \mathrm{H} & 1.01900 & 0.53800 & 1.60200 \\ \mathrm{H} & 1.22500 & 1.03800 & 3.29100 \\ \mathrm{H} & 2.36600 & -0.98100 & 2.44000 \\ \mathrm{H} & 1.24000 & 4.30600 & -2.80300 \\ \mathrm{H} & 1.93000 & 5.94100 & -2.95000 \\ \mathrm{H} & 1.42500 & 5.33500 & -1.35000 \\ \mathrm{H} & 5.46500 & 0.37200 & -3.01600 \\ \mathrm{H} & 5.15600 & -1.30600 & -0.46600 \\ \mathrm{H} & 6.57900 & -1.72200 & -3.17500 \\ \mathrm{H} & 5.75400 & -3.00700 & -2.28200 \\ \mathrm{H} & 7.97000 & -1.59100 & -1.28200\end{array}$

RSRS

\begin{tabular}{lrrr} 
O & -9.04600 & -1.61100 & -1.44300 \\
$C$ & -7.76200 & -1.33700 & -1.10500 \\
$C$ & -5.12000 & -0.75900 & -0.41200 \\
$C$ & -7.39600 & -0.04000 & -0.71400 \\
$C$ & -6.80200 & -2.33100 & -1.15200 \\
$C$ & -5.48400 & -2.04000 & -0.80700 \\
$C$ & -6.08600 & 0.24900 & -0.36800 \\
O & -8.42900 & 0.85300 & -0.72200 \\
$C$ & -8.14400 & 2.18700 & -0.34700 \\
$C$ & -3.69300 & -0.48800 & 0.00500 \\
$C$ & -3.40200 & -1.14500 & 1.36400 \\
$O$ & -2.04100 & -0.95400 & 1.75600 \\
$C$ & -4.20700 & -0.55300 & 2.51100 \\
\hline & -3.90000 & -1.19600 & 3.72900
\end{tabular}




\begin{tabular}{|c|c|c|c|}
\hline $\mathrm{O}$ & -3.50300 & 0.92500 & 0.07000 \\
\hline 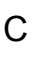 & -1.02300 & -1.50900 & 1.04000 \\
\hline ; & 1.19200 & -2.44400 & -0.42200 \\
\hline C & 0.25300 & -0.95000 & 1.24400 \\
\hline C & -1.16700 & -2.54800 & 0.12600 \\
\hline C & -0.07200 & -3.00500 & -0.60100 \\
\hline C & 1.33100 & -1.40900 & 0.50800 \\
\hline C & 2.33800 & -2.94200 & -1.19700 \\
\hline C & 3.62100 & -2.78600 & -0.86500 \\
\hline C & 4.75300 & -3.31800 & -1.68500 \\
\hline O & 5.48400 & -4.32600 & -0.98100 \\
\hline $\mathrm{O}$ & 0.47800 & 0.10200 & 2.07900 \\
\hline C & -0.11600 & 0.11400 & 3.37200 \\
\hline$C$ & -2.22800 & 1.43400 & 0.09200 \\
\hline$c$ & 0.28800 & 2.65100 & 0.16100 \\
\hline$c$ & -1.92000 & 2.36900 & 1.07000 \\
\hline$C$ & -1.27300 & 1.13200 & -0.89400 \\
\hline$C$ & -0.01700 & 1.72900 & -0.83500 \\
\hline C & -0.67600 & 2.98700 & 1.10500 \\
\hline $\mathrm{O}$ & -1.64800 & 0.26600 & -1.87100 \\
\hline C & -0.66200 & -0.14000 & -2.80600 \\
\hline C & 1.67200 & 3.24700 & 0.25600 \\
\hline $\mathrm{O}$ & 2.35700 & 3.10600 & -1.00800 \\
\hline C & 2.56000 & 2.54100 & 1.32200 \\
\hline C & 3.51600 & 1.75900 & 0.45400 \\
\hline C & 5.09500 & 0.72700 & -1.54700 \\
\hline C & 3.33900 & 2.17700 & -0.86000 \\
\hline C & 4.48300 & 0.82600 & 0.78500 \\
\hline C & 5.28900 & 0.29100 & -0.23200 \\
\hline C & 4.12500 & 1.66500 & -1.89300 \\
\hline $\mathrm{O}$ & 3.98100 & 1.99000 & -3.20500 \\
\hline C & 3.30100 & 3.54000 & 2.21400 \\
\hline
\end{tabular}




\begin{tabular}{|c|c|c|c|}
\hline 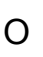 & 4.06300 & 4.46400 & 1.46700 \\
\hline 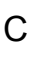 & 3.75400 & 3.36500 & -3.51000 \\
\hline ; & 6.34400 & -0.70100 & 0.03600 \\
\hline C & 6.42800 & -1.50200 & 1.09900 \\
\hline C & 7.52400 & -2.50900 & 1.29400 \\
\hline O & 7.01300 & -3.82100 & 1.40300 \\
\hline $\mathrm{H}$ & -9.55800 & -0.79700 & -1.35400 \\
\hline $\mathrm{H}$ & -7.10000 & -3.32400 & -1.46600 \\
\hline $\mathrm{H}$ & -4.73600 & -2.82500 & -0.86200 \\
\hline $\mathrm{H}$ & -5.78800 & 1.24700 & -0.07700 \\
\hline $\mathrm{H}$ & -7.40700 & 2.63100 & -1.02200 \\
\hline$H$ & -7.77200 & 2.23000 & 0.68100 \\
\hline$H$ & -9.08200 & 2.73100 & -0.42000 \\
\hline$H$ & -3.02000 & -0.91000 & -0.74100 \\
\hline $\mathrm{H}$ & -3.62400 & -2.21700 & 1.30200 \\
\hline $\mathrm{H}$ & -5.27000 & -0.70300 & 2.32400 \\
\hline $\mathrm{H}$ & -4.00700 & 0.52400 & 2.56600 \\
\hline $\mathrm{H}$ & -2.94500 & -1.15800 & 3.83600 \\
\hline $\mathrm{H}$ & -2.13900 & -2.99300 & -0.04100 \\
\hline $\mathrm{H}$ & -0.20800 & -3.80900 & -1.31500 \\
\hline $\mathrm{H}$ & 2.28200 & -0.90700 & 0.65100 \\
\hline $\mathrm{H}$ & 2.09400 & -3.49000 & -2.10600 \\
\hline $\mathrm{H}$ & 3.90300 & -2.28300 & 0.05700 \\
\hline$\pi$ & 5.47900 & -2.53100 & -1.89600 \\
\hline 11 & 4.38700 & -3.71400 & -2.63800 \\
\hline 11 & 4.86400 & -5.01800 & -0.72900 \\
\hline 11 & -0.22100 & -0.90200 & 3.76100 \\
\hline$\Pi$ & 0.56500 & 0.67400 & 4.01300 \\
\hline$\Pi$ & -1.08800 & 0.60800 & 3.34500 \\
\hline $\mathrm{H}$ & -2.68700 & 2.61000 & 1.79800 \\
\hline $\mathrm{r}$ & 0.73200 & 1.49500 & -1.57900 \\
\hline $\mathrm{H}$ & -0.45600 & 3.72000 & 1.87400 \\
\hline
\end{tabular}




$\begin{array}{lrrr}\mathrm{H} & 0.19200 & -0.59500 & -2.29600 \\ \mathrm{H} & -0.32700 & 0.70600 & -3.41300 \\ \mathrm{H} & -1.14500 & -0.87700 & -3.44400 \\ \mathrm{H} & 1.61400 & 4.31900 & 0.45700 \\ \mathrm{H} & 1.95100 & 1.87600 & 1.93900 \\ \mathrm{H} & 5.71300 & 0.33600 & -2.35000 \\ \mathrm{H} & 4.63300 & 0.53300 & 1.81800 \\ \mathrm{H} & 3.93000 & 2.99400 & 2.92800 \\ \mathrm{H} & 2.58000 & 4.13200 & 2.78200 \\ \mathrm{H} & 4.71900 & 3.97400 & 0.96000 \\ \mathrm{H} & 4.44900 & 3.99900 & -2.95000 \\ \mathrm{H} & 2.73100 & 3.66000 & -3.28000 \\ \mathrm{H} & 3.94500 & 3.46800 & -4.57600 \\ \mathrm{H} & 7.11100 & -0.78600 & -0.73300 \\ \mathrm{H} & 5.65900 & -1.48800 & 1.87000 \\ \mathrm{H} & 8.05600 & -2.31100 & 2.22900 \\ \mathrm{H} & 8.24900 & -2.42600 & 0.47400 \\ \mathrm{H} & 6.54200 & -4.01700 & 0.58000\end{array}$

RSSR

$\begin{array}{llll}\text { O } & -8.47100 & -0.71300 & 2.20100 \\ \text { C } & -7.29200 & -0.39100 & 1.61700 \\ \text { C } & -4.86100 & 0.25900 & 0.41700 \\ \text { C } & -6.61700 & -1.33300 & 0.82400 \\ \text { C } & -6.74100 & 0.86400 & 1.80600 \\ \text { C } & -5.52500 & 1.18800 & 1.20900 \\ \text { C } & -5.40900 & -1.01100 & 0.22500 \\ \text { O } & -7.25700 & -2.53500 & 0.72400 \\ \text { C } & -6.64400 & -3.53900 & -0.06200 \\ \text { C } & -3.56200 & 0.64700 & -0.25100 \\ \text { C } & -3.78000 & 1.61100 & -1.41800 \\ \text { O } & -2.52900 & 2.01300 & -2.00100\end{array}$




\begin{tabular}{|c|c|c|c|}
\hline C & -4.56100 & 1.00500 & -2.57100 \\
\hline 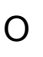 & -4.68400 & 1.93000 & -3.63000 \\
\hline O & -2.86900 & -0.48000 & -0.77600 \\
\hline C & -1.46100 & 2.26700 & -1.17800 \\
\hline ; & 0.77400 & 2.72300 & 0.45900 \\
\hline C & -1.53600 & 3.18500 & -0.12500 \\
\hline C & -0.27200 & 1.57800 & -1.40000 \\
\hline C & 0.83300 & 1.79900 & -0.59100 \\
\hline C & -0.43100 & 3.39100 & 0.68900 \\
\hline C & 1.93800 & 3.05000 & 1.30000 \\
\hline C & 3.21700 & 2.92800 & 0.94300 \\
\hline C & 4.36700 & 3.34900 & 1.81200 \\
\hline O & 5.27700 & 2.29100 & 2.05200 \\
\hline $\mathrm{O}$ & -2.70800 & 3.84200 & 0.15500 \\
\hline$c$ & -3.06300 & 4.82800 & -0.81400 \\
\hline$C$ & -1.79200 & -0.95100 & -0.08700 \\
\hline$C$ & 0.55200 & -1.84700 & 1.14200 \\
\hline C & -1.64700 & -0.85100 & 1.29200 \\
\hline C & -0.78000 & -1.55200 & -0.84900 \\
\hline C & 0.38100 & -1.99000 & -0.23300 \\
\hline C & -0.47400 & -1.29200 & 1.89800 \\
\hline $\mathrm{O}$ & -0.87500 & -1.62000 & -2.21200 \\
\hline C & -1.87500 & -2.50700 & -2.70000 \\
\hline C & 1.85200 & -2.22200 & 1.81100 \\
\hline $\mathrm{O}$ & 2.56200 & -3.20800 & 1.02900 \\
\hline C & 2.82200 & -1.01800 & 1.94200 \\
\hline C & 3.72100 & -1.24200 & 0.75400 \\
\hline C & 5.23200 & -2.24800 & -1.30900 \\
\hline C & 3.52600 & -2.55000 & 0.32100 \\
\hline C & 4.67000 & -0.42600 & 0.17100 \\
\hline C & 5.45300 & -0.93800 & -0.87800 \\
\hline C & 4.27400 & -3.08000 & -0.72600 \\
\hline
\end{tabular}




\begin{tabular}{|c|c|c|c|}
\hline 0 & 4.15400 & -4.36100 & -1.17300 \\
\hline $\mathrm{C}$ & 3.61700 & -1.10400 & 3.25200 \\
\hline$C$ & 4.68800 & -0.17800 & 3.30200 \\
\hline C & 2.83700 & -4.76800 & -1.53300 \\
\hline C & 6.49100 & -0.12500 & -1.53200 \\
\hline $\mathrm{C}$ & 6.94300 & 1.06200 & -1.11800 \\
\hline C & 8.00100 & 1.82700 & -1.85400 \\
\hline C & 9.12300 & 2.11400 & -1.03200 \\
\hline$\vdash$ & -8.68900 & -1.62100 & 1.95700 \\
\hline r & -7.27300 & 1.57200 & 2.42900 \\
\hline ト & -5.08800 & 2.16900 & 1.36800 \\
\hline 十 & -4.87500 & -1.72800 & -0.38500 \\
\hline ト & -6.54100 & -3.21000 & -1.10000 \\
\hline ⺊ & -7.29900 & -4.40500 & -0.01600 \\
\hline & -5.66000 & -3.79900 & 0.34000 \\
\hline & -2.92600 & 1.15300 & 0.48100 \\
\hline & -4.31300 & 2.48900 & -1.04100 \\
\hline & -5.57000 & 0.76000 & -2.23800 \\
\hline r & -4.05800 & 0.08800 & -2.90000 \\
\hline & -3.79100 & 2.18500 & -3.88400 \\
\hline r & -0.25500 & 0.84000 & -2.19500 \\
\hline & 1.74100 & 1.22900 & -0.76200 \\
\hline r & -0.52400 & 4.10700 & 1.49900 \\
\hline 十 & 1.71000 & 3.48500 & 2.27300 \\
\hline & 3.47800 & 2.53600 & -0.03800 \\
\hline & 3.99100 & 3.77100 & 2.75200 \\
\hline & 4.94500 & 4.12800 & 1.30700 \\
\hline r & 4.85900 & 1.63700 & 2.63100 \\
\hline & -3.99600 & 5.27400 & -0.47300 \\
\hline & -2.28600 & 5.59500 & -0.87100 \\
\hline & -3.20700 & 4.37600 & -1.80000 \\
\hline & -2.44500 & -0.42800 & 1.89000 \\
\hline
\end{tabular}




$\begin{array}{llll}\mathrm{H} & 1.15600 & -2.42000 & -0.85700 \\ \mathrm{H} & -0.36400 & -1.19700 & 2.97300 \\ \mathrm{H} & -1.81900 & -2.46600 & -3.78600 \\ \mathrm{H} & -2.86900 & -2.19600 & -2.37200 \\ \mathrm{H} & -1.67300 & -3.52800 & -2.36100 \\ \mathrm{H} & 1.65000 & -2.68100 & 2.78200 \\ \mathrm{H} & 2.29000 & -0.06400 & 1.89000 \\ \mathrm{H} & 5.81600 & -2.66300 & -2.12300 \\ \mathrm{H} & 4.80800 & 0.59000 & 0.52100 \\ \mathrm{H} & 3.99200 & -2.12600 & 3.38100 \\ \mathrm{H} & 2.96700 & -0.86900 & 4.09700 \\ \mathrm{H} & 5.37300 & -0.47700 & 2.69200 \\ \mathrm{H} & 2.43000 & -4.09500 & -2.29400 \\ \mathrm{H} & 2.17700 & -4.79000 & -0.66500 \\ \mathrm{H} & 2.93400 & -5.76800 & -1.95000 \\ \mathrm{H} & 6.91800 & -0.55500 & -2.43700 \\ \mathrm{H} & 6.57500 & 1.52000 & -0.20100 \\ \mathrm{H} & 7.62000 & 2.80200 & -2.16700 \\ \mathrm{H} & 8.30200 & 1.27900 & -2.75500 \\ \mathrm{H} & 9.42000 & 1.28900 & -0.63700\end{array}$

RSSS

$\begin{array}{llll}\text { O } & 8.88000 & -1.08700 & 1.11000 \\ \text { C } & 7.56100 & -0.99000 & 0.81500 \\ \text { C } & 4.85200 & -0.77700 & 0.19100 \\ \text { C } & 7.15300 & -0.38500 & -0.38800 \\ \text { C } & 6.61100 & -1.47700 & 1.69300 \\ \text { C } & 5.25500 & -1.36500 & 1.38100 \\ \text { C } & 5.81000 & -0.27900 & -0.70000 \\ \text { O } & 8.18400 & 0.05500 & -1.16700 \\ \text { C } & 7.84700 & 0.65800 & -2.40400 \\ \text { C } & 3.38900 & -0.65400 & -0.14200 \\ \text { C } & 2.89000 & -1.71500 & -1.14100\end{array}$




\begin{tabular}{|c|c|c|c|}
\hline $\mathrm{O}$ & 1.53900 & -1.42100 & -1.49000 \\
\hline 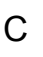 & 3.65000 & -1.80400 & -2.45700 \\
\hline 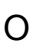 & 3.80000 & -0.57200 & -3.13000 \\
\hline $\mathrm{O}$ & 3.11500 & 0.64600 & -0.69100 \\
\hline C & 0.52700 & -1.74400 & -0.64200 \\
\hline C & -1.74600 & -2.48600 & 0.84500 \\
\hline C & -0.76400 & -1.33900 & -1.05800 \\
\hline C & 0.65900 & -2.44300 & 0.55000 \\
\hline C & -0.47000 & -2.80400 & 1.29100 \\
\hline C & -1.87500 & -1.71900 & -0.32400 \\
\hline C & -2.92900 & -2.97400 & 1.57400 \\
\hline C & -4.12800 & -3.19700 & 1.03500 \\
\hline C & -5.30100 & -3.76600 & 1.78000 \\
\hline $\mathrm{O}$ & -6.48700 & -3.01900 & 1.57500 \\
\hline $\mathrm{O}$ & -0.81900 & -0.59800 & -2.18900 \\
\hline$c$ & -2.05000 & 0.04900 & -2.46200 \\
\hline$C$ & 2.03800 & 1.29200 & -0.13900 \\
\hline$C$ & -0.20800 & 2.50400 & 0.98500 \\
\hline$C$ & 0.95700 & 1.62800 & -0.93800 \\
\hline C & 2.03100 & 1.62900 & 1.22200 \\
\hline C & 0.91000 & 2.23000 & 1.77400 \\
\hline C & -0.16200 & 2.23500 & -0.38000 \\
\hline $\mathrm{O}$ & 3.09300 & 1.29900 & 2.02300 \\
\hline C & 4.27200 & 2.07000 & 1.78400 \\
\hline C & -1.47700 & 2.98500 & 1.65000 \\
\hline $\mathrm{O}$ & -2.31700 & 3.64900 & 0.67600 \\
\hline C & -2.35800 & 1.82100 & 2.21500 \\
\hline C & -3.33900 & 1.63300 & 1.08500 \\
\hline C & -4.88800 & 1.79000 & -1.18900 \\
\hline C & -3.24800 & 2.74300 & 0.25500 \\
\hline C & -4.20400 & 0.59700 & 0.79900 \\
\hline C & -4.97400 & 0.66100 & -0.37200 \\
\hline
\end{tabular}




\begin{tabular}{|c|c|c|c|}
\hline C & -4.03000 & 2.85100 & -0.89500 \\
\hline 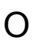 & -3.97000 & 3.88500 & -1.77600 \\
\hline 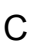 & -1.63200 & 0.54200 & 2.58900 \\
\hline O & -0.87000 & 0.80200 & 3.75900 \\
\hline C & -3.80700 & 5.19300 & -1.23100 \\
\hline C & -5.82700 & -0.49500 & -0.69400 \\
\hline C & -6.14400 & -0.91400 & -1.92000 \\
\hline C & -6.99600 & -2.12100 & -2.19700 \\
\hline O & -7.61900 & -2.66000 & -1.04000 \\
\hline $\mathrm{H}$ & 9.37700 & -0.69600 & 0.38200 \\
\hline $\mathrm{H}$ & 6.94400 & -1.92900 & 2.61800 \\
\hline $\mathrm{H}$ & 4.51100 & -1.71800 & 2.08800 \\
\hline$H$ & 5.47700 & 0.18200 & -1.62200 \\
\hline$H$ & 7.28400 & -0.03600 & -3.03500 \\
\hline $\mathrm{H}$ & 7.25500 & 1.56300 & -2.24400 \\
\hline $\mathrm{H}$ & 8.78900 & 0.91400 & -2.88300 \\
\hline$H$ & 2.81500 & -0.76700 & 0.78100 \\
\hline $\mathrm{H}$ & 2.96000 & -2.70200 & -0.66900 \\
\hline $\mathrm{H}$ & 4.65400 & -2.18800 & -2.26000 \\
\hline $\mathrm{H}$ & 3.12000 & -2.52700 & -3.08700 \\
\hline $\mathrm{H}$ & 3.03000 & -0.02700 & -2.93500 \\
\hline $\mathrm{H}$ & 1.63600 & -2.73700 & 0.91000 \\
\hline $\mathrm{H}$ & -0.34400 & -3.37500 & 2.20400 \\
\hline $\mathrm{H}$ & -2.86200 & -1.41300 & -0.64700 \\
\hline 11 & -2.77500 & -3.21500 & 2.62500 \\
\hline 11 & -4.29300 & -3.00300 & -0.02500 \\
\hline 11 & -5.45700 & -4.81000 & 1.47800 \\
\hline$\Pi$ & -5.10300 & -3.76000 & 2.85400 \\
\hline$\Pi$ & -6.78000 & -3.13800 & 0.66100 \\
\hline$\Pi$ & -2.82500 & -0.67100 & -2.74500 \\
\hline $\mathrm{H}$ & -1.86000 & 0.72300 & -3.29500 \\
\hline $\mathrm{H}$ & -2.38900 & 0.61800 & -1.59100 \\
\hline
\end{tabular}




\begin{tabular}{llll}
$\mathrm{H}$ & 0.97900 & 1.35300 & -1.98500 \\
$\mathrm{H}$ & 0.90700 & 2.44200 & 2.83700 \\
$\mathrm{H}$ & -1.00500 & 2.48300 & -1.01300 \\
$\mathrm{H}$ & 4.55500 & 2.03400 & 0.73000 \\
$\mathrm{H}$ & 4.10700 & 3.10600 & 2.09500 \\
$\mathrm{H}$ & 5.06200 & 1.62000 & 2.38400 \\
$\mathrm{H}$ & -1.24600 & 3.71800 & 2.42100 \\
$\mathrm{H}$ & -2.88400 & 2.18200 & 3.10600 \\
$\mathrm{H}$ & -5.50100 & 1.88000 & -2.07900 \\
$\mathrm{H}$ & -4.27500 & -0.27700 & 1.43900 \\
$\mathrm{H}$ & -2.37300 & -0.24300 & 2.78300 \\
$\mathrm{H}$ & -0.99800 & 0.20600 & 1.76100 \\
$\mathrm{H}$ & -0.22100 & 0.10100 & 3.86400 \\
$\mathrm{H}$ & -4.48300 & 5.33900 & -0.38400 \\
$\mathrm{H}$ & -2.78000 & 5.36300 & -0.90900 \\
$\mathrm{H}$ & -4.06900 & 5.88300 & -2.03100 \\
$\mathrm{H}$ & -6.16500 & -1.06700 & 0.16700 \\
$\mathrm{H}$ & -5.76900 & -0.39200 & -2.79700 \\
$\mathrm{H}$ & -6.38400 & -2.92900 & -2.60700 \\
$\mathrm{H}$ & -7.75000 & -1.88100 & -2.95600 \\
\hline & -8.23500 & -2.00500 & -0.69600
\end{tabular}

S.2. Optimized geometry for the RSSR scission products ( $298 \mathrm{~K}$ )

$\begin{array}{rrrr}\begin{array}{l}\boldsymbol{\alpha}-\mathbf{- O}-\mathbf{4} \\ \boldsymbol{\alpha}-\mathbf{O}\end{array} & & & \\ \text { Alpha_side } & & & \\ \text { O } & 3.41700 & -0.71200 & 1.50500 \\ \text { C } & 2.13500 & -1.02900 & 1.19000 \\ \text { C } & -0.51500 & -1.65700 & 0.47200 \\ \text { C } & 1.87600 & -2.06000 & 0.26300 \\ \text { C } & 1.07800 & -0.35200 & 1.77800 \\ \text { C } & -0.22700 & -0.66000 & 1.43300\end{array}$




\begin{tabular}{|c|c|c|c|}
\hline C & 0.58200 & -2.37700 & -0.08100 \\
\hline & 3.00600 & -2.65000 & -0.22500 \\
\hline & 2.84600 & -3.68000 & -1.18100 \\
\hline & -1.81800 & -1.90900 & -0.02100 \\
\hline C & -3.00200 & -1.02400 & 0.17200 \\
\hline & -3.07000 & 0.00600 & -0.87100 \\
\hline C & -4.30900 & -1.78700 & 0.02200 \\
\hline$c$ & -5.42700 & -0.93000 & 0.05300 \\
\hline C & -1.95000 & 0.80500 & -0.90300 \\
\hline$c$ & 0.53800 & 2.07400 & -0.72200 \\
\hline C & -1.73800 & 1.78600 & 0.07100 \\
\hline C & -0.96000 & 0.55300 & -1.84800 \\
\hline C & 0.27500 & 1.17500 & -1.76300 \\
\hline c & -0.50000 & 2.41800 & 0.14200 \\
\hline c & 1.89200 & 2.58200 & -0.45100 \\
\hline C & 3.01700 & 1.93800 & -0.76600 \\
\hline$c$ & 4.38700 & 2.40800 & -0.38500 \\
\hline$c$ & 5.08500 & 1.41700 & 0.35000 \\
\hline & -2.63700 & 2.05800 & 1.05700 \\
\hline$c$ & -4.00700 & 2.22600 & 0.69000 \\
\hline & 4.00400 & -1.29200 & 1.00100 \\
\hline & 1.29800 & 0.43000 & 2.49400 \\
\hline r & -1.03100 & -0.10200 & 1.89500 \\
\hline & 0.38000 & -3.15000 & -0.81200 \\
\hline r & 2.27800 & -4.51400 & -0.76000 \\
\hline r & 3.84800 & -4.01200 & -1.44000 \\
\hline r & 2.34000 & -3.30300 & -2.07500 \\
\hline r & -1.93100 & -2.70000 & -0.75800 \\
\hline & -2.98500 & -0.50500 & 1.13700 \\
\hline & -4.41400 & -2.49000 & 0.84900 \\
\hline & -4.27700 & -2.35600 & -0.91700 \\
\hline & -5.30100 & -0.28800 & -0.65400 \\
\hline
\end{tabular}




\begin{tabular}{|c|c|c|c|}
\hline $\mathrm{H}$ & -1.16100 & -0.19600 & -2.60400 \\
\hline $\mathrm{H}$ & 1.04700 & 0.94200 & -2.48600 \\
\hline $\mathrm{H}$ & -0.34200 & 3.12500 & 0.94900 \\
\hline $\mathrm{H}$ & 1.95800 & 3.51100 & 0.11200 \\
\hline $\mathrm{H}$ & 2.97300 & 0.97500 & -1.27200 \\
\hline $\mathrm{H}$ & 4.31400 & 3.34500 & 0.18200 \\
\hline $\mathrm{H}$ & 4.99300 & 2.60200 & -1.27400 \\
\hline $\mathrm{H}$ & 4.47600 & 1.03600 & 0.99400 \\
\hline $\mathrm{H}$ & -4.40900 & 2.99400 & 1.34900 \\
\hline $\mathrm{H}$ & -4.08800 & 2.55400 & -0.34900 \\
\hline $\mathrm{H}$ & -4.56200 & 1.29600 & 0.82700 \\
\hline \multicolumn{4}{|l|}{ O_side } \\
\hline $\mathrm{O}$ & -5.27000 & -1.24500 & -1.93400 \\
\hline C & -4.36100 & -0.56700 & -1.42100 \\
\hline$C$ & -2.29000 & 1.01000 & -0.25700 \\
\hline$C$ & -4.01600 & 0.74700 & -1.92800 \\
\hline$C$ & -3.58100 & -1.03300 & -0.27200 \\
\hline C & -2.57300 & -0.22800 & 0.27500 \\
\hline$C$ & -3.02900 & 1.49700 & -1.37400 \\
\hline O & -3.75800 & -2.20500 & 0.32600 \\
\hline$C$ & -4.71700 & -3.17400 & -0.11300 \\
\hline$C$ & -1.18400 & 1.87800 & 0.29000 \\
\hline O & -0.68500 & 1.35400 & 1.53200 \\
\hline$C$ & 0.04000 & 1.93900 & -0.65900 \\
\hline$C$ & 0.91600 & 0.87400 & -0.04900 \\
\hline$C$ & 2.20100 & -0.92700 & 1.57300 \\
\hline$C$ & 0.45000 & 0.64200 & 1.24200 \\
\hline$C$ & 2.01600 & 0.20600 & -0.55000 \\
\hline C & 2.67900 & -0.71500 & 0.28000 \\
\hline C & 1.08400 & -0.26200 & 2.08700 \\
\hline $\mathrm{O}$ & 0.68100 & -0.58500 & 3.34000 \\
\hline$C$ & 0.72000 & 3.31300 & -0.59100 \\
\hline
\end{tabular}




\begin{tabular}{|c|c|c|c|}
\hline O & 1.93100 & 3.34900 & -1.30900 \\
\hline C & 0.04900 & 0.42900 & 4.11600 \\
\hline C & 3.86300 & -1.46800 & -0.16500 \\
\hline C & 4.59000 & -1.23800 & -1.26000 \\
\hline C & 5.78500 & -2.05800 & -1.64000 \\
\hline O & 6.95400 & -1.26200 & -1.77200 \\
\hline $\mathrm{H}$ & -4.59900 & 1.09200 & -2.77400 \\
\hline $\mathrm{H}$ & -2.02700 & -0.61000 & 1.13000 \\
\hline $\mathrm{H}$ & -2.79500 & 2.47900 & -1.77500 \\
\hline $\mathrm{H}$ & -4.58400 & -4.01100 & 0.56800 \\
\hline $\mathrm{H}$ & -4.51800 & -3.47500 & -1.14000 \\
\hline $\mathrm{H}$ & -5.72700 & -2.77600 & -0.04700 \\
\hline $\mathrm{H}$ & -1.57600 & 2.87700 & 0.50400 \\
\hline $\mathrm{H}$ & -0.23400 & 1.71500 & -1.69200 \\
\hline $\mathrm{H}$ & 2.69100 & -1.63900 & 2.22800 \\
\hline $\mathrm{H}$ & 2.33000 & 0.36300 & -1.57500 \\
\hline $\mathrm{H}$ & 0.87300 & 3.58400 & 0.46200 \\
\hline $\mathrm{H}$ & 0.07100 & 4.06500 & -1.04400 \\
\hline $\mathrm{H}$ & 2.55800 & 2.76400 & -0.87100 \\
\hline $\mathrm{H}$ & -0.98400 & 0.58000 & 3.80400 \\
\hline $\mathrm{H}$ & 0.59500 & 1.37200 & 4.03200 \\
\hline $\mathrm{H}$ & 0.08000 & 0.07700 & 5.14500 \\
\hline $\mathrm{H}$ & 4.16200 & -2.28700 & 0.48700 \\
\hline $\mathrm{H}$ & 4.35600 & -0.41300 & -1.92900 \\
\hline $\mathrm{H}$ & 5.63900 & -2.51700 & -2.62100 \\
\hline $\mathrm{H}$ & 5.93400 & -2.86300 & -0.91100 \\
\hline $\mathrm{H}$ & 7.08600 & -0.79000 & -0.94600 \\
\hline $\mathrm{O}$ & -5.44400 & -2.16600 & 1.99700 \\
\hline C & -4.39200 & -1.62600 & 1.33500 \\
\hline C & -2.23500 & -0.51900 & -0.04000 \\
\hline
\end{tabular}




\begin{tabular}{|c|c|c|c|}
\hline C & -4.46900 & -1.40700 & -0.05100 \\
\hline C & -3.23500 & -1.29300 & 2.01700 \\
\hline & -2.15800 & -0.73800 & 1.33000 \\
\hline & -3.39800 & -0.85700 & -0.73500 \\
\hline & -5.65800 & -1.78400 & -0.60500 \\
\hline & -5.81700 & -1.59900 & -1.99900 \\
\hline & -1.06800 & 0.10800 & -0.77700 \\
\hline & -0.84900 & 1.58200 & -0.31700 \\
\hline$C$ & 0.38000 & 2.10700 & -0.81000 \\
\hline C & -1.92500 & 2.50300 & -0.86400 \\
\hline C & -1.70700 & 3.83200 & -0.44500 \\
\hline C & -1.12900 & 0.05700 & -2.13200 \\
\hline C & 1.53000 & 1.43800 & -0.48000 \\
\hline C & 3.91000 & 0.04800 & 0.06700 \\
\hline C & 1.91700 & 1.20700 & 0.84600 \\
\hline C & 2.34800 & 0.99700 & -1.51700 \\
\hline C & 3.52700 & 0.32000 & -1.25200 \\
\hline C & 3.09400 & 0.51200 & 1.10000 \\
\hline C & 5.13200 & -0.69900 & 0.40900 \\
\hline C & 5.89600 & -1.40200 & -0.42900 \\
\hline C & 7.12800 & -2.13800 & 0.00300 \\
\hline O & 7.06300 & -3.51700 & -0.32300 \\
\hline O & 1.15400 & 1.60900 & 1.91000 \\
\hline C & 1.04200 & 3.02400 & 2.06800 \\
\hline $\mathrm{H}$ & -6.14700 & -2.33100 & 1.35700 \\
\hline $\mathrm{H}$ & -3.19400 & -1.47700 & 3.08400 \\
\hline $\mathrm{H}$ & -1.25100 & -0.48300 & 1.87000 \\
\hline $\mathrm{H}$ & -3.43400 & -0.69400 & -1.80500 \\
\hline $\mathrm{H}$ & -5.73100 & -0.54100 & -2.26200 \\
\hline $\mathrm{H}$ & -6.81400 & -1.95700 & -2.24200 \\
\hline ト & -5.07200 & -2.17600 & -2.55400 \\
\hline & -0.12800 & -0.41200 & -0.50000 \\
\hline
\end{tabular}




$\begin{array}{lrrr}\mathrm{H} & -0.85700 & 1.59600 & 0.77500 \\ \mathrm{H} & -2.89700 & 2.19500 & -0.47700 \\ \mathrm{H} & -1.93700 & 2.42700 & -1.95900 \\ \mathrm{H} & -0.86000 & 4.10700 & -0.81000 \\ \mathrm{H} & 2.02200 & 1.19200 & -2.53200 \\ \mathrm{H} & 4.15000 & 0.00100 & -2.07700 \\ \mathrm{H} & 3.35900 & 0.33800 & 2.13800 \\ \mathrm{H} & 5.41500 & -0.66700 & 1.46000 \\ \mathrm{H} & 5.63800 & -1.49500 & -1.48100 \\ \mathrm{H} & 7.29600 & -1.99000 & 1.07700 \\ \mathrm{H} & 8.00400 & -1.75900 & -0.52800 \\ \mathrm{H} & 6.26800 & -3.88100 & 0.07600 \\ \mathrm{H} & 0.54000 & 3.18500 & 3.01900 \\ \mathrm{H} & 2.03600 & 3.47800 & 2.09300 \\ \mathrm{H} & 0.45100 & 3.46800 & 1.26300\end{array}$

4_side

$\begin{array}{llll}\text { C } & -4.41000 & -0.42400 & -1.75300 \\ \text { C } & -2.55000 & 0.92000 & -0.28200 \\ \text { C } & -4.23700 & 0.91400 & -2.01300 \\ \text { C } & -3.71300 & -1.13400 & -0.79900 \\ \text { C } & -2.76000 & -0.43200 & -0.04900 \\ \text { C } & -3.28600 & 1.59800 & -1.26000 \\ \text { O } & -3.88700 & -2.44800 & -0.54000 \\ \text { C } & -4.85900 & -3.10500 & -1.33600 \\ \text { C } & -1.49900 & 1.69400 & 0.48700 \\ \text { O } & -1.04000 & 0.94400 & 1.62800 \\ \text { C } & -0.23200 & 1.97900 & -0.35600 \\ \text { C } & 0.65900 & 0.84500 & 0.08100 \\ \text { C } & 1.93900 & -1.20000 & 1.38800 \\ \text { C } & 0.13700 & 0.34400 & 1.27100 \\ \text { C } & 1.81100 & 0.32600 & -0.47700 \\ \text { C } & 2.47200 & -0.71900 & 0.19200\end{array}$




\begin{tabular}{|c|c|c|c|}
\hline$C$ & 0.76900 & -0.68800 & 1.95700 \\
\hline $\mathrm{O}$ & 0.32000 & -1.26800 & 3.09700 \\
\hline$C$ & 0.38600 & 3.33200 & 0.02000 \\
\hline 0 & 1.63000 & 3.55000 & -0.60500 \\
\hline$C$ & -0.41000 & -0.45600 & 4.01200 \\
\hline$C$ & 3.70900 & -1.32900 & -0.32200 \\
\hline$C$ & 4.47700 & -0.86800 & -1.31100 \\
\hline$C$ & 5.72400 & -1.55900 & -1.77100 \\
\hline 0 & 6.86300 & -0.71500 & -1.67800 \\
\hline $\mathrm{H}$ & -4.82000 & 1.42300 & -2.77200 \\
\hline $\mathrm{H}$ & -2.20600 & -0.96800 & 0.71300 \\
\hline $\mathrm{H}$ & -3.11900 & 2.65700 & -1.43100 \\
\hline $\mathrm{H}$ & -4.87200 & -4.14100 & -1.00700 \\
\hline $\mathrm{H}$ & -4.59000 & -3.05300 & -2.39500 \\
\hline $\mathrm{H}$ & -5.84500 & -2.65400 & -1.19200 \\
\hline $\mathrm{H}$ & -1.93700 & 2.61600 & 0.87800 \\
\hline $\mathrm{H}$ & -0.44700 & 1.95800 & -1.42600 \\
\hline $\mathrm{H}$ & 2.42700 & -2.01100 & 1.91800 \\
\hline $\mathrm{H}$ & 2.16800 & 0.69500 & -1.43100 \\
\hline $\mathrm{H}$ & 0.47500 & 3.39300 & 1.11300 \\
\hline $\mathrm{H}$ & -0.27100 & 4.13900 & -0.31200 \\
\hline $\mathrm{H}$ & 2.25500 & 2.90400 & -0.26200 \\
\hline $\mathrm{H}$ & -1.43200 & -0.29300 & 3.67000 \\
\hline $\mathrm{H}$ & 0.08600 & 0.50800 & 4.14700 \\
\hline $\mathrm{H}$ & -0.41400 & -1.00200 & 4.95300 \\
\hline $\mathrm{H}$ & 4.01200 & -2.24800 & 0.17700 \\
\hline $\mathrm{H}$ & 4.23900 & 0.06100 & -1.82300 \\
\hline $\mathrm{H}$ & 5.65000 & -1.82200 & -2.82900 \\
\hline $\mathrm{H}$ & 5.87000 & -2.48600 & -1.20400 \\
\hline $\mathrm{H}$ & 6.92900 & -0.40500 & -0.77100 \\
\hline
\end{tabular}

B-O-4

1- $\alpha$ 
1_side

$\begin{array}{lrrr}\text { O } & -0.20600 & 2.21200 & -0.00000 \\ \text { C } & -0.56500 & 0.90300 & -0.00000 \\ \text { C } & -1.28000 & -1.71900 & 0.00000 \\ \text { C } & 0.42700 & -0.09400 & -0.00000 \\ \text { C } & -1.90500 & 0.55600 & -0.00000 \\ \text { C } & -2.28600 & -0.79200 & 0.00000 \\ \text { C } & 0.07000 & -1.44100 & -0.00000 \\ \text { O } & 1.70200 & 0.39200 & 0.00000 \\ \text { C } & 2.75800 & -0.54900 & 0.00000 \\ \text { H } & 0.75700 & 2.25900 & -0.00000 \\ \text { H } & -2.64300 & 1.35000 & 0.00000 \\ \text { H } & -3.33200 & -1.07200 & 0.00000 \\ \text { H } & 0.81400 & -2.22700 & -0.00000 \\ \text { H } & 2.71800 & -1.17700 & 0.89400 \\ \text { H } & 3.68000 & 0.02700 & 0.00000 \\ \text { H } & 2.71800 & -1.17700 & -0.89400\end{array}$

Alpha_side

$\begin{array}{llll}\text { C } & -4.89100 & 0.54500 & -0.45700 \\ \text { C } & -5.51400 & -0.27300 & 0.60700 \\ \text { O } & -4.51100 & -0.96800 & 1.40000 \\ \text { C } & -6.27200 & 0.55400 & 1.64300 \\ \text { O } & -6.77900 & -0.26500 & 2.67000 \\ \text { O } & -3.95200 & 1.42000 & -0.02100 \\ \text { C } & -3.39800 & -1.44400 & 0.75900 \\ \text { C } & -1.06500 & -2.41300 & -0.47200 \\ \text { C } & -3.48700 & -2.31900 & -0.32800 \\ \text { C } & -2.14100 & -1.04300 & 1.20800 \\ \text { C } & -0.98700 & -1.51900 & 0.60300 \\ \text { C } & -2.32800 & -2.78200 & -0.93800 \\ \text { C } & 0.12300 & -3.02100 & -1.09700 \\ \text { C } & 1.32700 & -3.15100 & -0.53600\end{array}$




\begin{tabular}{|c|c|c|c|}
\hline C & 2.46800 & -3.88900 & -1.17400 \\
\hline & 3.65300 & -3.11600 & -1.23100 \\
\hline & -4.70700 & -2.70300 & -0.82600 \\
\hline & -5.42100 & -3.59400 & 0.02900 \\
\hline & -2.73700 & 1.52800 & -0.64800 \\
\hline & -0.13600 & 1.74500 & -1.63500 \\
\hline C & -2.34200 & 0.75500 & -1.73300 \\
\hline c & -1.83900 & 2.43000 & -0.06600 \\
\hline C & -0.54800 & 2.52900 & -0.56300 \\
\hline$c$ & -1.04600 & 0.87000 & -2.22100 \\
\hline C & -2.18400 & 3.15300 & 1.04000 \\
\hline$c$ & -3.15100 & 4.17300 & 0.80800 \\
\hline$c$ & 1.28300 & 1.80500 & -2.15900 \\
\hline c & 2.05000 & 2.79800 & -1.44700 \\
\hline c & 2.04900 & 0.47400 & -1.95800 \\
\hline & 2.77600 & 0.76100 & -0.66900 \\
\hline C & 4.13700 & 1.86800 & 1.43900 \\
\hline & 2.76000 & 2.14000 & -0.48000 \\
\hline & 3.46200 & -0.07900 & 0.18500 \\
\hline & 4.16700 & 0.48300 & 1.26200 \\
\hline c & 3.44300 & 2.72500 & 0.58200 \\
\hline & 3.52600 & 4.06500 & 0.80600 \\
\hline & 3.04300 & 0.24800 & -3.10400 \\
\hline C & 3.94600 & -0.81100 & -2.84000 \\
\hline c & 2.30500 & 4.79500 & 0.75600 \\
\hline C & 4.93200 & -0.34800 & 2.20500 \\
\hline C & 5.19600 & -1.65100 & 2.07900 \\
\hline C & 5.98500 & -2.42100 & 3.09400 \\
\hline O & 7.13100 & -3.03600 & 2.52500 \\
\hline & -5.02600 & 0.38800 & -1.51800 \\
\hline & -6.18900 & -1.00200 & 0.15200 \\
\hline & -7.12500 & 1.03600 & 1.16300 \\
\hline
\end{tabular}




\begin{tabular}{|c|c|c|c|}
\hline 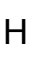 & -5.60000 & 1.32600 & 2.03600 \\
\hline 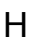 & -6.02800 & -0.72700 & 3.05700 \\
\hline 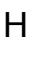 & -2.09700 & -0.32600 & 2.02000 \\
\hline I & -0.02100 & -1.17100 & 0.95200 \\
\hline 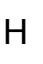 & -2.43500 & -3.46700 & -1.77300 \\
\hline $\mathrm{H}$ & -0.03800 & -3.46100 & -2.08100 \\
\hline 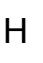 & 1.51700 & -2.75700 & 0.46100 \\
\hline 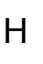 & 2.17500 & -4.24500 & -2.16900 \\
\hline 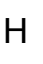 & 2.71400 & -4.76600 & -0.57000 \\
\hline 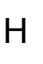 & 3.56900 & -2.45300 & -1.93200 \\
\hline $\mathrm{H}$ & -6.35300 & -3.83200 & -0.48200 \\
\hline $\mathrm{H}$ & -4.84300 & -4.50900 & 0.18600 \\
\hline $\mathrm{H}$ & -5.63500 & -3.12600 & 0.99400 \\
\hline $\mathrm{H}$ & -3.01100 & 0.02000 & -2.15900 \\
\hline $\mathrm{H}$ & 0.13200 & 3.22000 & -0.08300 \\
\hline $\mathrm{H}$ & -0.74000 & 0.24100 & -3.05100 \\
\hline $\mathrm{H}$ & -3.29800 & 4.67700 & 1.76200 \\
\hline $\mathrm{H}$ & -4.09700 & 3.75000 & 0.46700 \\
\hline $\mathrm{H}$ & -2.77500 & 4.88800 & 0.07000 \\
\hline $\mathrm{H}$ & 1.28000 & 2.11400 & -3.20700 \\
\hline $\mathrm{H}$ & 1.37100 & -0.37900 & -1.87300 \\
\hline $\mathrm{H}$ & 4.66700 & 2.32600 & 2.26700 \\
\hline $\mathrm{H}$ & 3.45400 & -1.15000 & 0.02100 \\
\hline $\mathrm{H}$ & 3.59200 & 1.17700 & -3.29800 \\
\hline $\mathrm{H}$ & 2.50600 & -0.02600 & -4.01400 \\
\hline $\mathrm{H}$ & 4.56500 & -0.51700 & -2.16100 \\
\hline $\mathrm{H}$ & 1.56800 & 4.34600 & 1.43000 \\
\hline 11 & 1.90600 & 4.83400 & -0.25900 \\
\hline 11 & 2.54200 & 5.80000 & 1.09900 \\
\hline 11 & 5.31300 & 0.17400 & 3.08200 \\
\hline II & 4.86500 & -2.21400 & 1.20800 \\
\hline $\mathrm{H}$ & 5.39000 & -3.24300 & 3.49800 \\
\hline
\end{tabular}




$$
\begin{array}{llll}
\mathrm{H} & 6.26200 & -1.76600 & 3.92900 \\
\mathrm{H} & 7.62900 & -2.36000 & 2.05900
\end{array}
$$

$\alpha-\beta$

Alpha_side

\begin{tabular}{llll} 
O & 8.96200 & 2.58200 & -0.16700 \\
C & 7.81100 & 1.86900 & -0.12500 \\
C & 5.43200 & 0.38100 & -0.03900 \\
C & 7.84300 & 0.46500 & -0.26600 \\
C & 6.59500 & 2.51100 & 0.05700 \\
C & 5.41500 & 1.78700 & 0.10000 \\
C & 6.68200 & -0.27100 & -0.22300 \\
O & 9.10200 & -0.03700 & -0.43800 \\
$C$ & 9.23300 & -1.43600 & -0.58900 \\
C & 4.25400 & -0.38400 & -0.00500 \\
O & 3.08000 & 0.24000 & 0.28400 \\
C & 1.90500 & -0.37200 & -0.04800 \\
C & -0.59000 & -1.47800 & -0.64500 \\
C & 1.77500 & -1.26400 & -1.10300 \\
C & 0.78100 & -0.02400 & 0.71900 \\
C & -0.45200 & -0.57400 & 0.41200 \\
C & 0.53000 & -1.81900 & -1.39100 \\
O & 0.87700 & 0.88600 & 1.73000 \\
C & 1.57700 & 0.42400 & 2.88200 \\
C & -1.94100 & -2.04000 & -1.01000 \\
O & -2.68200 & -2.39400 & 0.18200 \\
C & -2.84400 & -1.04200 & -1.77900 \\
C & -3.73900 & -0.53900 & -0.67500 \\
\hline & -5.27200 & -0.18700 & 1.57300 \\
\hline & -3.61000 & -1.41500 & 0.40000 \\
\hline
\end{tabular}




\begin{tabular}{|c|c|c|c|}
\hline $\mathrm{O}$ & -4.32200 & -2.05300 & 2.65000 \\
\hline C & -3.66200 & -1.77200 & -2.85300 \\
\hline O & -4.60900 & -0.93700 & -3.47700 \\
\hline C & -3.06600 & -2.63300 & 2.99200 \\
\hline C & -6.40400 & 1.78700 & 0.62400 \\
\hline C & -6.81400 & 2.60700 & -0.34500 \\
\hline C & -7.82300 & 3.69300 & -0.12900 \\
\hline $\mathrm{O}$ & -8.95100 & 3.54500 & -0.98000 \\
\hline & 9.69100 & 1.96500 & -0.30100 \\
\hline & 6.59600 & 3.58900 & 0.16200 \\
\hline & 4.46800 & 2.29100 & 0.23900 \\
\hline & 6.70400 & -1.34900 & -0.32800 \\
\hline & 8.86400 & -1.95900 & 0.29800 \\
\hline & 10.29500 & -1.63200 & -0.71300 \\
\hline $\mathrm{H}$ & 8.69000 & -1.78300 & -1.47300 \\
\hline & 4.23600 & -1.46500 & -0.07400 \\
\hline & 2.63800 & -1.50400 & -1.71200 \\
\hline & -1.30200 & -0.27700 & 1.01600 \\
\hline & 0.43900 & -2.52000 & -2.21400 \\
\hline & 1.54700 & 1.23700 & 3.60400 \\
\hline & 2.61500 & 0.18500 & 2.64300 \\
\hline & 1.07700 & -0.45600 & 3.29800 \\
\hline & -1.80700 & -2.96700 & -1.57400 \\
\hline & -2.25800 & -0.24700 & -2.24200 \\
\hline & -5.86100 & -0.05900 & 2.47500 \\
\hline & -4.67500 & 1.21500 & -1.47000 \\
\hline & -4.14500 & -2.64900 & -2.39900 \\
\hline & -2.99400 & -2.12400 & -3.64200 \\
\hline & -5.28100 & -0.70700 & -2.82800 \\
\hline & -2.27000 & -1.88500 & 2.93500 \\
\hline & -2.82200 & -3.46700 & 2.33500 \\
\hline & -3.17100 & -2.98000 & 4.01800 \\
\hline
\end{tabular}




$\begin{array}{llll}\mathrm{H} & -6.83100 & 1.91400 & 1.61800 \\ \mathrm{H} & -6.44200 & 2.50700 & -1.36200 \\ \mathrm{H} & -7.40000 & 4.66700 & -0.38200 \\ \mathrm{H} & -8.12400 & 3.72000 & 0.92500 \\ \mathrm{H} & -9.31800 & 2.66900 & -0.83700\end{array}$

Beta_side

\begin{tabular}{|c|c|c|c|}
\hline C & -3.21400 & -0.53300 & 0.90000 \\
\hline 0 & -2.44300 & -0.88600 & -0.17800 \\
\hline i & -4.62600 & -0.96400 & 0.74500 \\
\hline כ & -5.21600 & -0.44700 & -0.43900 \\
\hline & -1.12000 & -0.51500 & -0.17800 \\
\hline ; & 1.61300 & 0.11100 & -0.23100 \\
\hline ; & -0.70000 & 0.78200 & 0.14000 \\
\hline C & -0.18400 & -1.47200 & -0.55900 \\
\hline C & 1.16500 & -1.16500 & -0.59300 \\
\hline C & 0.66000 & 1.07000 & 0.11700 \\
\hline C & 3.03700 & 0.48200 & -0.21200 \\
\hline c & 4.07600 & -0.35300 & -0.24500 \\
\hline C & 5.50200 & 0.10500 & -0.22100 \\
\hline $\mathrm{O}$ & 6.22500 & -0.46600 & 0.85900 \\
\hline 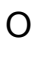 & -1.57200 & 1.76700 & 0.50500 \\
\hline$c$ & -2.44500 & 2.20900 & -0.53500 \\
\hline $\mathrm{H}$ & -2.71100 & -0.41500 & 1.85100 \\
\hline $\mathrm{H}$ & -5.21200 & -0.59000 & 1.58500 \\
\hline $\mathrm{H}$ & -4.70100 & -2.06400 & 0.74500 \\
\hline $\mathrm{H}$ & -4.69900 & -0.77100 & -1.18300 \\
\hline $\mathrm{H}$ & -0.54400 & -2.45900 & -0.82300 \\
\hline $\mathrm{H}$ & 1.87300 & -1.92200 & -0.90700 \\
\hline ונו & 0.96000 & 2.07800 & 0.38300 \\
\hline $\mathrm{H}$ & 3.23700 & 1.55100 & -0.15100 \\
\hline 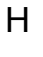 & 3.92900 & -1.43100 & -0.26600 \\
\hline & 5.54300 & 1.20100 & -0.19000 \\
\hline
\end{tabular}




\begin{tabular}{|c|c|c|c|}
\hline $\mathrm{H}$ & 6.02600 & -0.22400 & -1.12100 \\
\hline $\mathrm{H}$ & 5.76300 & -0.25100 & 1.67400 \\
\hline $\mathrm{H}$ & -3.00800 & 3.04300 & -0.12300 \\
\hline $\mathrm{H}$ & -1.86000 & 2.54600 & -1.39600 \\
\hline $\mathrm{H}$ & -3.14000 & 1.42200 & -0.83600 \\
\hline \multicolumn{4}{|l|}{$\beta-O$} \\
\hline \multicolumn{4}{|l|}{ Beta_side } \\
\hline $\mathrm{O}$ & 7.36800 & -2.50200 & -0.77500 \\
\hline$C$ & 6.52600 & -1.44600 & -0.87300 \\
\hline$C$ & 4.77200 & 0.71600 & -1.07100 \\
\hline$C$ & 5.68000 & -1.11700 & 0.19900 \\
\hline$C$ & 6.48100 & -0.69400 & -2.03400 \\
\hline$C$ & 5.60200 & 0.38300 & -2.13300 \\
\hline $\mathrm{C}$ & 4.81500 & -0.04000 & 0.10400 \\
\hline $\mathrm{O}$ & 5.79900 & -1.94200 & 1.27800 \\
\hline$C$ & 4.93400 & -1.70900 & 2.37500 \\
\hline$C$ & 3.85700 & 1.92900 & -1.17100 \\
\hline $\mathrm{C}$ & 4.58300 & 3.17700 & -0.77500 \\
\hline C & 4.64800 & 3.55600 & 0.66800 \\
\hline $\mathrm{O}$ & 3.45600 & 4.18700 & 1.12900 \\
\hline $\mathrm{O}$ & 2.76000 & 1.83600 & -0.26100 \\
\hline C & 1.80400 & 0.88100 & -0.41600 \\
\hline C & -0.29700 & -0.95800 & -0.51400 \\
\hline $\mathrm{C}$ & 1.75600 & -0.06000 & -1.43800 \\
\hline$C$ & 0.80200 & 0.89500 & 0.56600 \\
\hline$C$ & -0.23800 & -0.01700 & 0.51500 \\
\hline C & 0.70400 & -0.97200 & -1.47700 \\
\hline $\mathrm{O}$ & 0.83700 & 1.84900 & 1.54800 \\
\hline C & 1.75800 & 1.57100 & 2.59700 \\
\hline$C$ & -1.45300 & -1.92400 & -0.63400 \\
\hline $\mathrm{O}$ & -2.07700 & -2.13000 & 0.65200 \\
\hline C & -2.58000 & -1.40800 & -1.56300 \\
\hline
\end{tabular}




\begin{tabular}{|c|c|c|c|}
\hline C & -3.53700 & -0.82400 & -0.55600 \\
\hline C & -5.01500 & -0.16900 & 1.66400 \\
\hline C & -3.20100 & -1.35200 & 0.68700 \\
\hline C & -4.59900 & 0.04700 & -0.70300 \\
\hline C & -5.36100 & 0.38200 & 0.42900 \\
\hline C & -3.93800 & -1.04300 & 1.82500 \\
\hline $\mathrm{O}$ & -3.64900 & -1.48100 & 3.07900 \\
\hline C & -3.23600 & -2.56600 & -2.32800 \\
\hline $\mathrm{O}$ & -4.36900 & -2.15700 & -3.05800 \\
\hline C & -3.22600 & -2.83500 & 3.20700 \\
\hline C & -6.51400 & 1.29400 & 0.36300 \\
\hline C & -7.13200 & 1.72100 & -0.74000 \\
\hline C & -8.30200 & 2.65700 & -0.71600 \\
\hline $\mathrm{O}$ & -9.43900 & 2.10200 & -1.36100 \\
\hline $\mathrm{H}$ & 7.24800 & -2.90200 & 0.09400 \\
\hline $\mathrm{H}$ & 7.13400 & -0.96900 & -2.85300 \\
\hline$H$ & 5.56900 & 0.96400 & -3.04900 \\
\hline$H$ & 4.14800 & 0.20600 & 0.91900 \\
\hline$H$ & 5.11600 & -0.72100 & 2.80900 \\
\hline H & 5.16100 & -2.47800 & 3.11000 \\
\hline$H$ & 3.88800 & -1.78900 & 2.06700 \\
\hline$H$ & 3.47700 & 2.01300 & -2.19600 \\
\hline$H$ & 5.07400 & 3.76100 & -1.54100 \\
\hline $\mathrm{H}$ & 5.45200 & 4.27200 & 0.83900 \\
\hline $\mathrm{H}$ & 4.86000 & 2.66300 & 1.27600 \\
\hline $\mathrm{H}$ & 2.71700 & 3.61300 & 0.89400 \\
\hline $\mathrm{H}$ & 2.53700 & -0.10100 & -2.18600 \\
\hline $\mathrm{H}$ & -0.99700 & 0.02400 & 1.28800 \\
\hline $\mathrm{H}$ & 0.67600 & -1.70800 & -2.27500 \\
\hline $\mathrm{H}$ & 1.67900 & 2.39300 & 3.30600 \\
\hline $\mathrm{H}$ & 2.78400 & 1.52800 & 2.21600 \\
\hline $\mathrm{H}$ & 1.50100 & 0.62800 & 3.08800 \\
\hline
\end{tabular}




$\begin{array}{llll}\mathrm{H} & -1.08400 & -2.90000 & -0.96000 \\ \mathrm{H} & -2.20600 & -0.66500 & -2.27100 \\ \mathrm{H} & -5.58300 & 0.08200 & 2.55300 \\ \mathrm{H} & -4.80900 & 0.49100 & -1.66900 \\ \mathrm{H} & -3.48800 & -3.36500 & -1.61700 \\ \mathrm{H} & -2.52900 & -2.97000 & -3.05600 \\ \mathrm{H} & -5.04600 & -1.88100 & -2.43300 \\ \mathrm{H} & -2.19900 & -2.96500 & 2.86600 \\ \mathrm{H} & -3.88600 & -3.49500 & 2.63500 \\ \mathrm{H} & -3.30200 & -3.07200 & 4.26600 \\ \mathrm{H} & -6.88700 & 1.63900 & 1.32600 \\ \mathrm{H} & -6.82200 & 1.38200 & -1.72600 \\ \mathrm{H} & -8.07400 & 3.56900 & -1.27300 \\ \mathrm{H} & -8.53200 & 2.94200 & 0.31700 \\ \mathrm{H} & -9.63700 & 1.26100 & -0.94100\end{array}$

O_side

$\begin{array}{lrrr}\text { O } & -3.31800 & 1.38800 & -0.10000 \\ \text { C } & -2.16100 & 0.94800 & -0.02900 \\ \text { C } & 0.53200 & -0.02900 & 0.13400 \\ \text { C } & -1.85600 & -0.48800 & -0.00300 \\ \text { C } & -1.01100 & 1.83500 & 0.03300 \\ \text { C } & 0.26200 & 1.37500 & 0.11100 \\ \text { C } & -0.53600 & -0.92000 & 0.07500 \\ \text { C } & 1.88600 & -0.56300 & 0.21300 \\ \text { C } & 3.02400 & 0.14000 & 0.28200 \\ \text { C } & 4.37900 & -0.49600 & 0.35800 \\ \text { O } & 5.23300 & -0.04000 & -0.67900 \\ \text { O } & -2.77600 & -1.44900 & -0.05200 \\ \text { C } & -4.18100 & -1.18400 & -0.13300 \\ \text { H } & -1.23900 & 2.89400 & 0.01400 \\ \text { H } & 1.08400 & 2.07900 & 0.15600 \\ \text { H } & -0.36000 & -1.98900 & 0.09200\end{array}$




$\begin{array}{llll}\mathrm{H} & 1.95600 & -1.65000 & 0.22000 \\ \mathrm{H} & 3.02100 & 1.22600 & 0.26800 \\ \mathrm{H} & 4.28400 & -1.58800 & 0.34700 \\ \mathrm{H} & 4.87800 & -0.21300 & 1.28800 \\ \mathrm{H} & 4.81700 & -0.24200 & -1.52100 \\ \mathrm{H} & -4.63900 & -2.17000 & -0.15400 \\ \mathrm{H} & -4.52000 & -0.61900 & 0.73400 \\ \mathrm{H} & -4.41900 & -0.62800 & -1.03700\end{array}$

$\beta-\gamma$

Beta_side

$\begin{array}{llll}\text { O } & -9.33300 & 1.34500 & 0.46400 \\ \text { C } & -8.00300 & 1.12700 & 0.31700 \\ \text { C } & -5.27000 & 0.66900 & 0.02500 \\ \text { C } & -7.51700 & -0.17800 & 0.15000 \\ \text { C } & -7.11700 & 2.19000 & 0.33800 \\ \text { C } & -5.75100 & 1.96100 & 0.19100 \\ \text { C } & -6.15800 & -0.40900 & 0.00200 \\ \text { O } & -8.49000 & -1.13800 & 0.15600 \\ \text { C } & -8.08000 & -2.48300 & 0.00700 \\ \text { C } & -3.79400 & 0.44900 & -0.19700 \\ \text { C } & -3.48300 & 0.62200 & -1.64300 \\ \text { O } & -2.20400 & 0.71700 & -2.11000 \\ \text { O } & -3.45900 & -0.89200 & 0.25000 \\ \text { C } & -1.21800 & 1.31600 & -1.36400 \\ \text { C } & 0.89900 & 2.43300 & 0.09000 \\ \text { C } & -1.40400 & 2.52200 & -0.67600 \\ \text { C } & 0.02700 & 0.69800 & -1.35100 \\ \text { C } & 1.07700 & 1.25000 & -0.63700 \\ \text { C } & -0.35500 & 3.04600 & 0.06800 \\ \text { C } & 1.99800 & 3.05000 & 0.85400 \\ \text { C } & 3.29000 & 2.96300 & 0.53600 \\ \text { C } & 4.39500 & 3.58100 & 1.34200\end{array}$




\begin{tabular}{|c|c|c|c|}
\hline 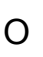 & 5.31700 & 2.60600 & 1.80300 \\
\hline 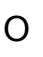 & -2.60900 & 3.17500 & -0.68400 \\
\hline C & -2.93500 & 3.77800 & -1.93600 \\
\hline C & -2.16000 & -1.10200 & 0.62200 \\
\hline C & 0.47600 & -1.65200 & 1.39400 \\
\hline C & -1.56700 & -0.39100 & 1.66400 \\
\hline C & -1.42300 & -2.08800 & -0.04000 \\
\hline C & -0.11900 & -2.36600 & 0.36000 \\
\hline C & -0.25900 & -0.65600 & 2.03900 \\
\hline $\mathrm{O}$ & -1.94700 & -2.76100 & -1.10800 \\
\hline C & -2.96800 & -3.69300 & -0.77100 \\
\hline C & 1.87000 & -1.98700 & 1.88200 \\
\hline $\mathrm{O}$ & 2.48100 & -2.97600 & 1.02800 \\
\hline$C$ & 2.84600 & -0.78200 & 1.91600 \\
\hline$c$ & 3.69900 & -1.05400 & 0.70400 \\
\hline$C$ & 5.06500 & -2.09500 & -1.43900 \\
\hline$C$ & 3.42100 & -2.34400 & 0.26400 \\
\hline$C$ & 4.66800 & -0.27900 & 0.09700 \\
\hline C & 5.37300 & -0.80800 & -0.99700 \\
\hline C & 4.09300 & -2.89100 & -0.82600 \\
\hline $\mathrm{O}$ & 3.90800 & -4.14900 & -1.30400 \\
\hline C & 3.67900 & -0.78500 & 3.20300 \\
\hline 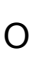 & 4.70800 & 0.18700 & 3.18600 \\
\hline 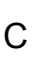 & 2.56700 & -4.61800 & -1.38500 \\
\hline C & 6.41200 & -0.03300 & -1.69400 \\
\hline C & 6.89900 & 1.15400 & -1.32200 \\
\hline C & 7.95500 & 1.87700 & -2.10300 \\
\hline $\mathrm{O}$ & 9.10600 & 2.15200 & -1.31900 \\
\hline $\mathrm{H}$ & -9.78000 & 0.49000 & 0.43100 \\
\hline $\mathrm{H}$ & -7.51200 & 3.18900 & 0.47600 \\
\hline $\mathrm{H}$ & -5.05100 & 2.78900 & 0.20700 \\
\hline & -5.76800 & -1.41100 & -0.11300 \\
\hline
\end{tabular}




\begin{tabular}{|c|c|c|c|}
\hline 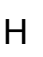 & -7.57600 & -2.63200 & -0.95300 \\
\hline 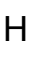 & -8.98500 & -3.08400 & 0.04100 \\
\hline 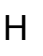 & -7.41100 & -2.77800 & 0.82100 \\
\hline I & -3.23200 & 1.16400 & 0.40500 \\
\hline 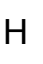 & -4.14100 & 0.19500 & -2.38900 \\
\hline $\mathrm{H}$ & 0.13700 & -0.23600 & -1.88900 \\
\hline 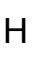 & 2.03500 & 0.73900 & -0.62100 \\
\hline 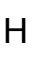 & -0.53200 & 3.97000 & 0.60800 \\
\hline 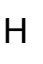 & 1.70800 & 3.64300 & 1.72000 \\
\hline $\mathrm{H}$ & 3.59400 & 2.41700 & -0.35500 \\
\hline $\mathrm{H}$ & 3.97700 & 4.15800 & 2.17500 \\
\hline $\mathrm{H}$ & 4.97800 & 4.26600 & 0.72100 \\
\hline $\mathrm{H}$ & 4.87000 & 2.01800 & 2.42900 \\
\hline $\mathrm{H}$ & -3.94100 & 4.18100 & -1.83100 \\
\hline $\mathrm{H}$ & -2.23100 & 4.58400 & -2.15800 \\
\hline $\mathrm{H}$ & -2.92200 & 3.04100 & -2.74300 \\
\hline $\mathrm{H}$ & -2.14300 & 0.36800 & 2.18100 \\
\hline $\mathrm{H}$ & 0.42400 & -3.14600 & -0.15700 \\
\hline $\mathrm{H}$ & 0.18500 & -0.09100 & 2.85200 \\
\hline $\mathrm{H}$ & -3.30200 & -4.13600 & -1.70800 \\
\hline $\mathrm{H}$ & -3.80300 & -3.19200 & -0.27700 \\
\hline $\mathrm{H}$ & -2.56600 & -4.47400 & -0.11800 \\
\hline $\mathrm{H}$ & 1.79300 & -2.45200 & 2.86900 \\
\hline $\mathrm{H}$ & 2.31600 & 0.17100 & 1.82900 \\
\hline $\mathrm{H}$ & 5.58400 & -2.52300 & -2.28900 \\
\hline $\mathrm{H}$ & 4.87500 & 0.72100 & 0.45600 \\
\hline $\mathrm{H}$ & 4.10000 & -1.78500 & 3.36300 \\
\hline 11 & 3.04100 & -0.54500 & 4.05700 \\
\hline 11 & 5.40300 & -0.11800 & 2.59100 \\
\hline 11 & 1.93300 & -3.87100 & -1.87300 \\
\hline $\mathrm{H}$ & 2.16700 & -4.85200 & -0.39800 \\
\hline $\mathrm{H}$ & 2.60100 & -5.51800 & -1.99500 \\
\hline
\end{tabular}




$\begin{array}{llll}\mathrm{H} & 6.80500 & -0.49200 & -2.60000 \\ \mathrm{H} & 6.56400 & 1.64700 & -0.41000 \\ \mathrm{H} & 7.59000 & 2.85400 & -2.42800 \\ \mathrm{H} & 8.21700 & 1.30100 & -3.00000 \\ \mathrm{H} & 9.39600 & 1.32800 & -0.91900\end{array}$

\section{Y-O}

Gamma_side

\begin{tabular}{lrrr} 
O & -8.60900 & -0.47100 & 1.99100 \\
C & -7.41600 & -0.19100 & 1.41300 \\
C & -4.95600 & 0.37100 & 0.22900 \\
C & -6.76700 & -1.16000 & 0.63100 \\
C & -6.82700 & 1.04700 & 1.59800 \\
C & -5.59500 & 1.32700 & 1.00800 \\
C & -5.54600 & -0.88200 & 0.03900 \\
O & -7.44600 & -2.34100 & 0.53400 \\
C & -6.84600 & -3.37800 & -0.22000 \\
C & -3.64300 & 0.70400 & -0.43600 \\
C & -3.83600 & 1.60500 & -1.67300 \\
O & -2.57100 & 1.96000 & -2.25000 \\
C & -4.62500 & 0.95500 & -2.74800 \\
O & -2.95900 & -0.45200 & -0.89200 \\
C & -1.52200 & 2.24500 & -1.41600 \\
C & 0.69000 & 2.76800 & 0.23800 \\
C & -1.60000 & 3.22600 & -0.42100 \\
C & -0.33800 & 1.52900 & -1.57100 \\
C & 0.75500 & 1.78300 & -0.75400 \\
C & -0.50900 & 3.46600 & 0.40300 \\
C & 1.84200 & 3.12900 & 1.08200 \\
C & 3.12600 & 2.96700 & 0.75900 \\
C & 4.26400 & 3.42700 & 1.62500 \\
O & 5.15400 & 2.37300 & 1.94900 \\
\hline & & & \\
\hline
\end{tabular}




\begin{tabular}{|c|c|c|c|}
\hline $\mathrm{O}$ & -2.76700 & 3.91900 & -0.20600 \\
\hline C & -3.06800 & 4.86000 & -1.23500 \\
\hline C & -1.90700 & -0.91600 & -0.16000 \\
\hline C & 0.39800 & -1.76900 & 1.16400 \\
\hline C & -1.79300 & -0.75600 & 1.21700 \\
\hline C & -0.88800 & -1.56600 & -0.87300 \\
\hline C & 0.25600 & -1.97900 & -0.20400 \\
\hline C & -0.64000 & -1.17300 & 1.87300 \\
\hline $\mathrm{O}$ & -0.91600 & -1.72400 & -2.22500 \\
\hline C & -2.11700 & -2.20000 & -2.83000 \\
\hline C & 1.68100 & -2.11600 & 1.87800 \\
\hline $\mathrm{O}$ & 2.39800 & -3.15000 & 1.16900 \\
\hline C & 2.66000 & -0.91400 & 1.96300 \\
\hline C & 3.58500 & -1.21400 & 0.81200 \\
\hline C & 5.13400 & -2.35100 & -1.15200 \\
\hline C & 3.38600 & -2.54200 & 0.44900 \\
\hline C & 4.55800 & -0.44300 & 0.20800 \\
\hline C & 5.36000 & -1.02000 & -0.79000 \\
\hline C & 4.15200 & -3.13800 & -0.54800 \\
\hline O & 4.02600 & -4.44000 & -0.92600 \\
\hline C & 3.42100 & -0.93000 & 3.29500 \\
\hline $\mathrm{O}$ & 4.50100 & -0.01300 & 3.31800 \\
\hline C & 2.71300 & -4.84800 & -1.29900 \\
\hline C & 6.42300 & -0.25700 & -1.46300 \\
\hline C & 6.87700 & 0.94800 & -1.10900 \\
\hline C & 7.96100 & 1.65900 & -1.86000 \\
\hline $\mathrm{O}$ & 9.06500 & 1.98300 & -1.02800 \\
\hline r & -8.85000 & -1.37600 & 1.75800 \\
\hline ⺊ & -7.33900 & 1.77600 & 2.21300 \\
\hline ⺊ & -5.12800 & 2.29400 & 1.16400 \\
\hline 1 & -5.03000 & -1.61600 & -0.56700 \\
\hline r & -6.71500 & -3.07400 & -1.26200 \\
\hline
\end{tabular}




\begin{tabular}{|c|c|c|c|}
\hline $\mathrm{H}$ & -7.52700 & -4.22400 & -0.16800 \\
\hline $\mathrm{H}$ & -5.87800 & -3.65700 & 0.20600 \\
\hline $\mathrm{H}$ & -3.01300 & 1.24700 & 0.27300 \\
\hline 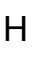 & -4.34700 & 2.50900 & -1.31200 \\
\hline $\mathrm{H}$ & -5.62300 & 0.59900 & -2.53600 \\
\hline $\mathrm{H}$ & -4.26900 & 1.01200 & -3.76600 \\
\hline $\mathrm{H}$ & -0.31300 & 0.74600 & -2.32100 \\
\hline $\mathrm{H}$ & 1.65700 & 1.19000 & -0.87200 \\
\hline $\mathrm{H}$ & -0.60800 & 4.23000 & 1.16700 \\
\hline $\mathrm{H}$ & 1.60200 & 3.62700 & 2.02000 \\
\hline $\mathrm{H}$ & 3.40100 & 2.51100 & -0.19000 \\
\hline $\mathrm{H}$ & 3.87500 & 3.91100 & 2.52800 \\
\hline $\mathrm{H}$ & 4.86300 & 4.16400 & 1.08500 \\
\hline $\mathrm{H}$ & 4.71200 & 1.75800 & 2.55200 \\
\hline $\mathrm{H}$ & -4.01400 & 5.32500 & -0.96300 \\
\hline $\mathrm{H}$ & -2.28600 & 5.62200 & -1.28900 \\
\hline $\mathrm{H}$ & -3.16100 & 4.36300 & -2.20500 \\
\hline $\mathrm{H}$ & -2.60300 & -0.29700 & 1.77100 \\
\hline $\mathrm{H}$ & 1.03800 & -2.44900 & -0.78700 \\
\hline $\mathrm{H}$ & -0.55500 & -1.02900 & 2.94500 \\
\hline $\mathrm{H}$ & -1.81000 & -2.71900 & -3.73700 \\
\hline $\mathrm{H}$ & -2.78800 & -1.37500 & -3.07300 \\
\hline $\mathrm{H}$ & -2.62800 & -2.90100 & -2.16400 \\
\hline $\mathrm{H}$ & 1.45600 & -2.51800 & 2.86900 \\
\hline $\mathrm{H}$ & 2.13800 & 0.03900 & 1.84200 \\
\hline $\mathrm{H}$ & 5.73200 & -2.81700 & -1.92800 \\
\hline $\mathrm{H}$ & 4.69900 & 0.59000 & 0.50300 \\
\hline $\mathrm{H}$ & 3.78200 & -1.94700 & 3.49400 \\
\hline $\mathrm{H}$ & 2.75300 & -0.64000 & 4.10900 \\
\hline $\mathrm{r}$ & 5.19600 & -0.35400 & 2.74300 \\
\hline $\mathrm{H}$ & 2.33700 & -4.21400 & -2.10800 \\
\hline $\mathrm{H}$ & 2.02900 & -4.81000 & -0.44900 \\
\hline
\end{tabular}




$\begin{array}{llll}\mathrm{H} & 2.80500 & -5.87200 & -1.65500 \\ \mathrm{H} & 6.86900 & -0.74400 & -2.32900 \\ \mathrm{H} & 6.49000 & 1.46400 & -0.23100 \\ \mathrm{H} & 7.59900 & 2.61600 & -2.24200 \\ \mathrm{H} & 8.28000 & 1.05400 & -2.71900 \\ \mathrm{H} & 9.33800 & 1.18300 & -0.57200\end{array}$

\section{0-4}

O_side

$\begin{array}{lrrr}\text { O } & 5.71200 & -3.86100 & 0.57400 \\ \text { C } & 5.35000 & -2.59600 & 0.26000 \\ \text { C } & 4.58500 & 0.00900 & -0.39300 \\ \text { C } & 4.27500 & -1.98300 & 0.92600 \\ \text { C } & 6.02600 & -1.90200 & -0.72700 \\ \text { C } & 5.63900 & -0.60300 & -1.05600 \\ \text { C } & 3.90100 & -0.68900 & 0.60700 \\ \text { O } & 3.67500 & -2.77100 & 1.86200 \\ \text { C } & 2.54500 & -2.24200 & 2.53100 \\ \text { C } & 4.20200 & 1.43600 & -0.73300 \\ \text { C } & 5.14500 & 2.46200 & -0.09200 \\ \text { O } & 4.99300 & 3.71500 & -0.62000 \\ \text { C } & 5.06900 & 2.52700 & 1.44500 \\ \text { O } & 6.03900 & 3.41100 & 1.94400 \\ \text { O } & 2.90800 & 1.77800 & -0.25800 \\ \text { C } & 1.81800 & 1.21200 & -0.84400 \\ \text { C } & -0.56500 & 0.18600 & -1.91300 \\ \text { C } & 1.85500 & 0.34500 & -1.93100 \\ \text { C } & 0.57800 & 1.56100 & -0.28200 \\ \text { C } & -0.59300 & 1.05300 & -0.81700 \\ \text { C } & 0.66700 & -0.15800 & -2.45600 \\ \text { O } & 0.51200 & 2.43700 & 0.76400 \\ \text { C } & 0.91500 & 1.88900 & 2.01100 \\ \text { C } & -1.82100 & -0.45500 & -2.44900\end{array}$




\begin{tabular}{|c|c|c|c|}
\hline $\mathrm{O}$ & -2.10300 & -1.65500 & -1.67000 \\
\hline C & -3.10300 & 0.39600 & -2.33500 \\
\hline 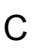 & -3.66700 & -0.09900 & -1.02600 \\
\hline C & -4.37000 & -1.50400 & 1.22200 \\
\hline C & -3.03300 & -1.30600 & -0.73300 \\
\hline C & -4.65600 & 0.41500 & -0.21100 \\
\hline C & -5.03100 & -0.31100 & 0.93400 \\
\hline C & -3.35500 & -2.02400 & 0.41400 \\
\hline O & -2.78500 & -3.19300 & 0.80100 \\
\hline C & -4.06500 & 0.08500 & -3.49100 \\
\hline O & -5.31600 & 0.71400 & -3.33500 \\
\hline C & -1.42800 & -3.41600 & 0.43100 \\
\hline$C$ & -6.08400 & 0.14900 & 1.85400 \\
\hline$C$ & -6.91000 & 1.18300 & 1.67800 \\
\hline$c$ & -7.95900 & 1.57300 & 2.67400 \\
\hline O & -9.26000 & 1.56700 & 2.10400 \\
\hline $\mathrm{H}$ & 5.12500 & -4.17900 & 1.27200 \\
\hline $\mathrm{H}$ & 6.84600 & -2.39400 & -1.23600 \\
\hline $\mathrm{H}$ & 6.16700 & -0.07400 & -1.84300 \\
\hline $\mathrm{H}$ & 3.06800 & -0.21600 & 1.11100 \\
\hline $\mathrm{H}$ & 2.81700 & -1.35900 & 3.11700 \\
\hline $\mathrm{H}$ & 2.19000 & -3.02600 & 3.19700 \\
\hline $\mathrm{H}$ & 1.76000 & -1.98000 & 1.81600 \\
\hline $\mathrm{H}$ & 4.24800 & 1.59500 & -1.81700 \\
\hline $\mathrm{H}$ & 6.18700 & 2.18400 & -0.34100 \\
\hline 11 & 5.27600 & 1.54100 & 1.86200 \\
\hline 11 & 4.05600 & 2.83100 & 1.73000 \\
\hline 11 & 5.91800 & 4.25500 & 1.49500 \\
\hline 11 & 2.79900 & 0.04400 & -2.36500 \\
\hline 11 & -1.52500 & 1.35800 & -0.35300 \\
\hline $\mathrm{H}$ & 0.71100 & -0.83900 & -3.29900 \\
\hline $\mathrm{H}$ & 0.76500 & 2.66700 & 2.75700 \\
\hline
\end{tabular}




$\begin{array}{llll}\mathrm{H} & 1.97100 & 1.60600 & 1.99200 \\ \mathrm{H} & 0.29800 & 1.01900 & 2.26100 \\ \mathrm{H} & -1.65100 & -0.80300 & -3.47000 \\ \mathrm{H} & -2.88400 & 1.46500 & -2.32400 \\ \mathrm{H} & -4.62500 & -2.06800 & 2.11300 \\ \mathrm{H} & -5.10800 & 1.37300 & -0.44100 \\ \mathrm{H} & -4.17600 & -1.00400 & -3.58000 \\ \mathrm{H} & -5.76000 & 0.31700 & -2.57900 \\ \mathrm{H} & -0.83400 & -2.51200 & 0.59300 \\ \mathrm{H} & -1.34500 & -3.71200 & -0.61400 \\ \mathrm{H} & -1.07300 & -4.21700 & 1.07800 \\ \mathrm{H} & -6.18400 & -0.43500 & 2.76800 \\ \mathrm{H} & -6.87300 & 1.78700 & 0.77500 \\ \mathrm{H} & -7.80200 & 2.59800 & 3.01600 \\ \mathrm{H} & -7.90700 & 0.91500 & 3.55000 \\ \mathrm{H} & -9.41600 & 0.69500 & 1.73100\end{array}$

4_side

$\begin{array}{llll}\text { C } & -2.31000 & 1.08400 & -0.00600 \\ \text { C } & 0.22500 & 0.04400 & 0.01800 \\ \text { C } & -2.18400 & -0.28800 & -0.02400 \\ \text { C } & -1.25300 & 1.96300 & 0.01300 \\ \text { C } & 0.03400 & 1.43400 & 0.02300 \\ \text { C } & -0.88300 & -0.80200 & -0.01600 \\ \text { C } & 1.56800 & -0.56400 & 0.04100 \\ \text { C } & 2.70900 & 0.04200 & 0.37200 \\ \text { C } & 4.04000 & -0.64700 & 0.35600 \\ \text { O } & 4.95400 & -0.00900 & -0.52400 \\ \text { O } & -3.21900 & -1.15700 & -0.05000 \\ \text { C } & -4.50800 & -0.56900 & -0.06000 \\ \text { H } & -1.41200 & 3.03500 & 0.01100 \\ \text { H } & 0.88600 & 2.10300 & 0.01700\end{array}$




$\begin{array}{llll}\mathrm{H} & -0.76000 & -1.88000 & -0.02800 \\ \mathrm{H} & 1.60300 & -1.62100 & -0.21800 \\ \mathrm{H} & 2.72500 & 1.09000 & 0.65900 \\ \mathrm{H} & 3.91200 & -1.70500 & 0.09500 \\ \mathrm{H} & 4.50900 & -0.59800 & 1.34100 \\ \mathrm{H} & 4.55400 & 0.01700 & -1.39800 \\ \mathrm{H} & -5.21900 & -1.39200 & -0.08000 \\ \mathrm{H} & -4.66400 & 0.03600 & 0.83800 \\ \mathrm{H} & -4.64000 & 0.06000 & -0.94500\end{array}$

B-5

$\alpha-P h$

Alpha_side

$\begin{array}{lrrr}\text { C } & -3.11100 & 1.32100 & -0.54200 \\ \text { O } & -3.05100 & 0.02800 & -0.06600 \\ \text { C } & -1.74500 & 1.93800 & -0.59200 \\ \text { C } & -0.88700 & 0.70700 & -0.42100 \\ \text { C } & 0.15900 & -1.76900 & 0.13400 \\ \text { C } & -1.72800 & -0.35000 & -0.07700 \\ \text { C } & 0.48200 & 0.53800 & -0.49900 \\ \text { C } & 1.02100 & -0.72600 & -0.20300 \\ \text { C } & -1.22900 & -1.61900 & 0.19900 \\ \text { O } & -1.94800 & -2.71100 & 0.54700 \\ \text { C } & -1.52700 & 2.94100 & 0.56500 \\ \text { O } & -0.21100 & 3.44400 & 0.60200 \\ \text { C } & -3.32000 & -2.76900 & 0.16800 \\ \text { C } & 2.46700 & -0.99800 & -0.24600 \\ \text { C } & 3.44700 & -0.10000 & -0.36600 \\ \text { C } & 4.89900 & -0.46900 & -0.39900 \\ \text { O } & 5.63200 & 0.17500 & 0.63400 \\ \text { H } & -4.04200 & 1.82700 & -0.32800 \\ \text { H } & -1.54300 & 2.45700 & -1.53400 \\ \text { H } & 0.55400 & -2.75600 & 0.34900\end{array}$




$\begin{array}{llll}\mathrm{H} & 1.11600 & 1.35400 & -0.82300 \\ \mathrm{H} & -1.79600 & 2.45500 & 1.51100 \\ \mathrm{H} & -2.18500 & 3.80000 & 0.42300 \\ \mathrm{H} & 0.37000 & 2.74400 & 0.91500 \\ \mathrm{H} & -3.43900 & -2.50800 & -0.88600 \\ \mathrm{H} & -3.92700 & -2.10000 & 0.77700 \\ \mathrm{H} & -3.62400 & -3.80100 & 0.33000 \\ \mathrm{H} & 2.74200 & -2.04900 & -0.16400 \\ \mathrm{H} & 3.23300 & 0.96400 & -0.42400 \\ \mathrm{H} & 5.36000 & -0.13100 & -1.33000 \\ \mathrm{H} & 5.01100 & -1.55800 & -0.34400 \\ \mathrm{H} & 5.22100 & -0.04900 & 1.47300\end{array}$

Phenyl_side

$\begin{array}{llll}\text { O } & 6.07000 & -1.22800 & -2.09300 \\ \text { C } & 4.88500 & -0.77500 & -1.61800 \\ \text { C } & 2.44400 & 0.14700 & -0.63500 \\ \text { C } & 4.30400 & -1.37200 & -0.48800 \\ \text { C } & 4.23700 & 0.27200 & -2.24900 \\ \text { C } & 3.01400 & 0.72900 & -1.76000 \\ \text { C } & 3.09200 & -0.91300 & 0.00300 \\ \text { O } & 5.03300 & -2.40100 & 0.03300 \\ \text { C } & 4.51100 & -3.06100 & 1.17100 \\ \text { C } & 1.13400 & 0.68200 & -0.09700 \\ \text { C } & 1.32900 & 2.01500 & 0.63400 \\ \text { O } & 0.11000 & 2.54900 & 1.18700 \\ \text { C } & 2.27600 & 1.91600 & 1.82100 \\ \text { O } & 2.37300 & 3.15200 & 2.49300 \\ \text { O } & 0.51800 & -0.22500 & 0.80900 \\ \text { C } & -1.09300 & 2.16500 & 0.65600 \\ \text { C } & -3.45100 & 1.08700 & -0.40300 \\ \text { C } & -1.38900 & 2.32800 & -0.70000 \\ \text { C } & -2.03200 & 1.57700 & 1.49500\end{array}$




\begin{tabular}{|c|c|c|c|}
\hline C & -3.20200 & 1.05200 & 0.97400 \\
\hline C & -2.55000 & 1.77300 & -1.22100 \\
\hline C & -4.56700 & 0.35700 & -1.02500 \\
\hline C & -5.28300 & -0.60600 & -0.44300 \\
\hline C & -6.30000 & -1.43900 & -1.15900 \\
\hline O & -5.95000 & -2.81600 & -1.10600 \\
\hline O & -0.48700 & 2.92800 & -1.54600 \\
\hline C & -0.29800 & 4.32100 & -1.30000 \\
\hline C & -0.43400 & -1.07000 & 0.30900 \\
\hline C & -2.48600 & -2.65500 & -0.53700 \\
\hline C & -0.54100 & -1.41100 & -1.03700 \\
\hline C & -1.35300 & -1.58600 & 1.24300 \\
\hline C & -2.40400 & -2.39100 & 0.80900 \\
\hline C & -1.59900 & -2.21400 & -1.48200 \\
\hline O & -1.27900 & -1.24500 & 2.56200 \\
\hline C & -0.14800 & -1.76500 & 3.25400 \\
\hline $\mathrm{H}$ & 6.36100 & -1.95500 & -1.53000 \\
\hline $\mathrm{H}$ & 4.69700 & 0.71200 & -3.12500 \\
\hline $\mathrm{H}$ & 2.50000 & 1.54000 & -2.26700 \\
\hline $\mathrm{H}$ & 2.62900 & -1.36800 & 0.86900 \\
\hline $\mathrm{H}$ & 4.41000 & -2.36600 & 2.01000 \\
\hline $\mathrm{H}$ & 5.22700 & -3.83900 & 1.42600 \\
\hline $\mathrm{H}$ & 3.54000 & -3.51200 & 0.94700 \\
\hline $\mathrm{H}$ & 0.46500 & 0.86100 & -0.93900 \\
\hline $\mathrm{H}$ & 1.72700 & 2.73400 & -0.09200 \\
\hline $\mathrm{H}$ & 3.27700 & 1.66000 & 1.47200 \\
\hline $\mathrm{H}$ & 1.91900 & 1.12400 & 2.49200 \\
\hline $\mathrm{H}$ & 1.47500 & 3.42300 & 2.71100 \\
\hline $\mathrm{H}$ & -1.78700 & 1.46800 & 2.54300 \\
\hline $\mathrm{H}$ & -3.89900 & 0.56300 & 1.64300 \\
\hline $\mathrm{H}$ & -2.71600 & 1.84900 & -2.29100 \\
\hline & -4.75800 & 0.58800 & -2.07100 \\
\hline
\end{tabular}




$\begin{array}{llll}\mathrm{H} & -5.08600 & -0.89800 & 0.58600 \\ \mathrm{H} & -6.40500 & -1.09700 & -2.19500 \\ \mathrm{H} & -7.27800 & -1.36900 & -0.67800 \\ \mathrm{H} & -5.01900 & -2.89500 & -1.34100 \\ \mathrm{H} & 0.42600 & 4.66800 & -2.03400 \\ \mathrm{H} & -1.24200 & 4.85700 & -1.43300 \\ \mathrm{H} & 0.08300 & 4.49300 & -0.29000 \\ \mathrm{H} & 0.19300 & -1.05300 & -1.74900 \\ \mathrm{H} & -3.12100 & -2.75700 & 1.53500 \\ \mathrm{H} & -1.69100 & -2.46300 & -2.53200 \\ \mathrm{H} & -0.25200 & -1.44900 & 4.29100 \\ \mathrm{H} & 0.78000 & -1.36600 & 2.84000 \\ \mathrm{H} & -0.14400 & -2.85700 & 3.20300\end{array}$

$\beta-5$

$\begin{array}{lrrr}\text { O } & -8.67400 & 0.49200 & -2.23000 \\ \text { C } & -7.46600 & 0.24900 & -1.66600 \\ \text { C } & -4.97800 & -0.24000 & -0.50800 \\ \text { C } & -6.88600 & 1.19400 & -0.80600 \\ \text { C } & -6.79400 & -0.92900 & -1.94200 \\ \text { C } & -5.55000 & -1.17300 & -1.36400 \\ \text { C } & -5.64900 & 0.95200 & -0.22800 \\ \text { O } & -7.64200 & 2.31500 & -0.62200 \\ \text { C } & -7.13200 & 3.31400 & 0.24000 \\ \text { C } & -3.64500 & -0.54500 & 0.13700 \\ \text { C } & -3.77100 & -1.60600 & 1.23100 \\ \text { O } & -2.48600 & -1.95900 & 1.76900 \\ \text { C } & -4.57300 & -1.14600 & 2.43600 \\ \text { O } & -4.62200 & -2.15800 & 3.41900 \\ \text { O } & -3.07000 & 0.61400 & 0.73300 \\ \text { C } & -1.40900 & -2.09600 & 0.93400 \\ \text { C } & 0.87800 & -2.34300 & -0.68500 \\ \text { C } & -1.43500 & -2.90500 & -0.20700\end{array}$




\begin{tabular}{|c|c|c|c|}
\hline C & -0.24200 & -1.40300 & 1.24800 \\
\hline ; & 0.88500 & -1.52100 & 0.44800 \\
\hline C & -0.30600 & -3.00800 & -1.00900 \\
\hline C & 2.08100 & -2.59100 & -1.50000 \\
\hline C & 3.34200 & -2.47100 & -1.08000 \\
\hline C & 4.53600 & -2.91300 & -1.87600 \\
\hline $\mathrm{O}$ & 5.63200 & -2.02000 & -1.78500 \\
\hline O & -2.58200 & -3.56800 & -0.57200 \\
\hline C & -2.89100 & -4.67700 & 0.27000 \\
\hline C & -1.92500 & 1.12200 & 0.19300 \\
\hline C & 0.53500 & 2.07300 & -0.73000 \\
\hline C & -1.62200 & 1.06700 & -1.16300 \\
\hline C & -1.01500 & 1.70600 & 1.08300 \\
\hline$C$ & 0.20100 & 2.18500 & 0.61600 \\
\hline C & -0.39100 & 1.52600 & -1.61500 \\
\hline $\mathrm{O}$ & -1.25700 & 1.71300 & 2.42900 \\
\hline$C$ & -2.32100 & 2.55900 & 2.84600 \\
\hline C & 1.87700 & 2.52100 & -1.26700 \\
\hline $\mathrm{O}$ & 2.67800 & 3.16800 & -0.23800 \\
\hline C & 2.60300 & 1.40400 & -1.95200 \\
\hline$C$ & 3.30000 & 1.18800 & 1.03100 \\
\hline$C$ & 5.91800 & 1.81600 & 0.78000 \\
\hline C & 3.59300 & 2.37600 & 0.39500 \\
\hline C & 4.20800 & 0.26900 & 1.47900 \\
\hline C & 5.57100 & 0.55700 & 1.28300 \\
\hline C & 4.95400 & 2.72400 & 0.33900 \\
\hline $\mathrm{O}$ & 5.36500 & 3.86700 & -0.27600 \\
\hline C & 3.92100 & 1.60800 & -2.62200 \\
\hline $\mathrm{O}$ & 4.90000 & 0.66300 & -2.18400 \\
\hline C & 4.90400 & 5.07800 & 0.32400 \\
\hline C & 6.62100 & -0.46200 & 1.43800 \\
\hline C & 6.39900 & -1.77400 & 1.37500 \\
\hline
\end{tabular}




\begin{tabular}{|c|c|c|c|}
\hline C & 7.47000 & -2.81000 & 1.22700 \\
\hline 0 & 7.27800 & -3.52500 & 0.02000 \\
\hline & -8.98000 & 1.35300 & -1.92100 \\
\hline & -7.25400 & -1.64100 & -2.61600 \\
\hline - & -5.01700 & -2.09200 & -1.59000 \\
\hline | & -5.18600 & 1.67400 & 0.43200 \\
\hline y & -6.99400 & 2.92000 & 1.25200 \\
\hline I & -7.87200 & 4.11000 & 0.25600 \\
\hline - & -6.18100 & 3.70300 & -0.13500 \\
\hline$H$ & -2.96900 & -0.94000 & -0.62500 \\
\hline$H$ & -4.24800 & -2.49000 & 0.79600 \\
\hline $\mathrm{H}$ & -5.60100 & -0.94200 & 2.13300 \\
\hline $\mathrm{H}$ & -4.12500 & -0.22800 & 2.83300 \\
\hline $\mathrm{H}$ & -3.71100 & -2.37000 & 3.64700 \\
\hline $\mathrm{H}$ & -0.25700 & -0.75100 & 2.11400 \\
\hline $\mathrm{H}$ & 1.77200 & -0.94900 & 0.69500 \\
\hline $\mathrm{H}$ & -0.36300 & -3.64900 & -1.88300 \\
\hline $\mathrm{H}$ & 1.90100 & -2.98500 & -2.49900 \\
\hline $\mathrm{H}$ & 3.55400 & -2.11000 & -0.07600 \\
\hline $\mathrm{H}$ & 4.25100 & -3.08600 & -2.92000 \\
\hline $\mathrm{H}$ & 4.90300 & -3.86100 & -1.46900 \\
\hline $\mathrm{H}$ & 5.36100 & -1.14500 & -2.10400 \\
\hline $\mathrm{H}$ & -3.81900 & -5.10400 & -0.10600 \\
\hline 1 & -2.09300 & -5.42300 & 0.21700 \\
\hline $\mathrm{H}$ & -3.02500 & -4.35700 & 1.30800 \\
\hline 1 & -2.33800 & 0.65300 & -1.86200 \\
\hline 1 & 0.89200 & 2.61500 & 1.33100 \\
\hline 1 & -0.15100 & 1.45200 & -2.67000 \\
\hline 1 & -2.37800 & 2.46900 & 3.93000 \\
\hline T & -3.26800 & 2.24600 & 2.40100 \\
\hline $\mathrm{H}$ & -2.10600 & 3.59700 & 2.57500 \\
\hline H & 1.71600 & 3.34200 & -1.97700 \\
\hline
\end{tabular}




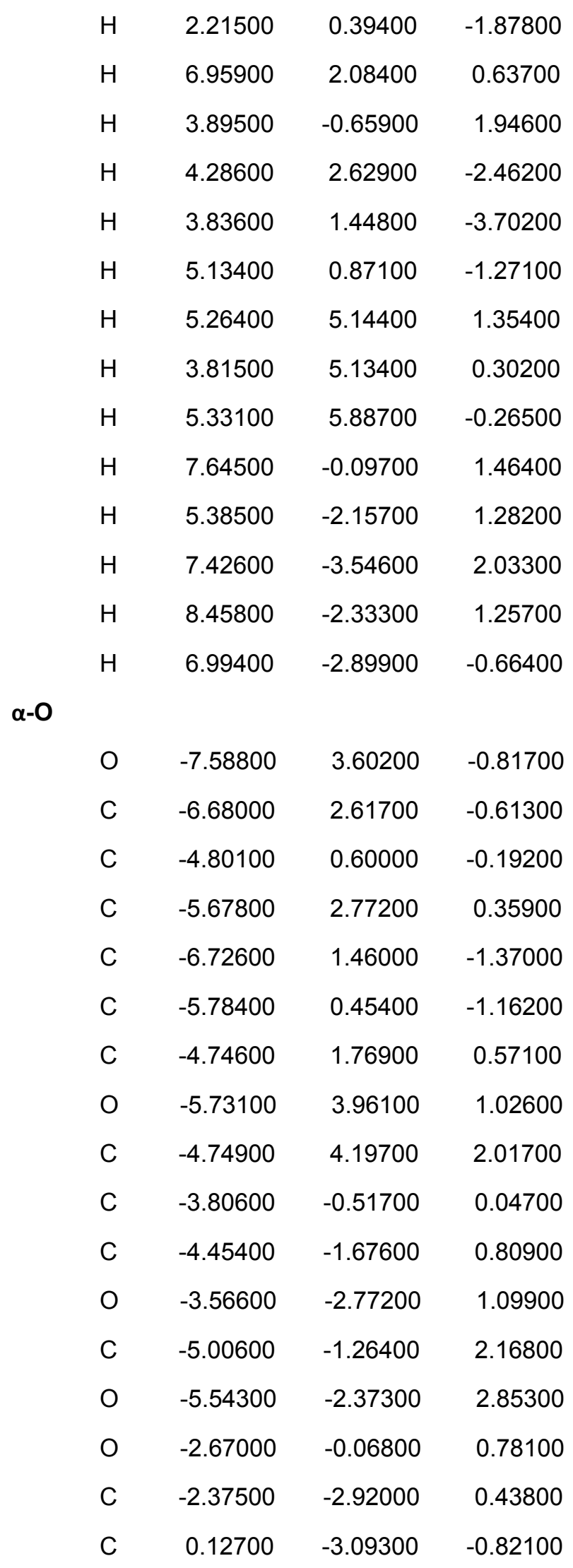




\begin{tabular}{|c|c|c|c|}
\hline C & -2.29600 & -3.03000 & -0.95300 \\
\hline 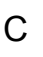 & -1.20900 & -2.97800 & 1.19300 \\
\hline ; & 0.02400 & -3.06800 & 0.57500 \\
\hline C & -1.05100 & -3.08100 & -1.57100 \\
\hline C & 1.43400 & -3.09000 & -1.50300 \\
\hline C & 2.60400 & -3.41200 & -0.94900 \\
\hline C & 3.91900 & -3.37500 & -1.66700 \\
\hline $\mathrm{O}$ & 4.85800 & -2.61500 & -0.91300 \\
\hline O & -3.43800 & -3.01500 & -1.71900 \\
\hline C & -4.23200 & -4.19200 & -1.58200 \\
\hline C & -1.57000 & 0.26100 & 0.03600 \\
\hline C & 0.78500 & 0.60400 & -1.48400 \\
\hline C & -1.64200 & 0.71000 & -1.28600 \\
\hline$C$ & -0.30900 & 0.10100 & 0.63900 \\
\hline$c$ & 0.83900 & 0.24100 & -0.11400 \\
\hline$C$ & -0.49000 & 0.88700 & -2.02600 \\
\hline $\mathrm{O}$ & -0.20100 & -0.27200 & 1.95200 \\
\hline$C$ & -0.69300 & 0.68800 & 2.87800 \\
\hline C & 1.93500 & 0.67400 & -2.30400 \\
\hline $\mathrm{O}$ & 3.67900 & 3.01000 & -2.18200 \\
\hline C & 3.28900 & 0.20000 & -1.87900 \\
\hline C & 3.98000 & 1.07800 & -0.85500 \\
\hline C & 5.26200 & 2.71400 & 1.05200 \\
\hline C & 4.07100 & 2.51400 & -1.11400 \\
\hline C & 4.55200 & 0.53000 & 0.25300 \\
\hline C & 5.22500 & 1.33100 & 1.22400 \\
\hline C & 4.68900 & 3.32300 & -0.05600 \\
\hline O & 4.79600 & 4.65000 & -0.12000 \\
\hline C & 4.18800 & 0.01000 & -3.11000 \\
\hline $\mathrm{O}$ & 5.45400 & -0.55100 & -2.77500 \\
\hline C & 4.01800 & 5.44200 & -1.02400 \\
\hline C & 5.86100 & 0.74100 & 2.39400 \\
\hline
\end{tabular}




\begin{tabular}{|c|c|c|c|}
\hline C & 5.99600 & -0.56900 & 2.64700 \\
\hline C & 6.66200 & -1.09500 & 3.88200 \\
\hline 0 & 7.70300 & -2.00800 & 3.57600 \\
\hline-1 & -7.39200 & 4.32200 & -0.20500 \\
\hline $\mathrm{H}$ & -7.49900 & 1.36600 & -2.12300 \\
\hline $\mathrm{H}$ & -5.81400 & -0.44500 & -1.76900 \\
\hline $\mathrm{H}$ & -3.96500 & 1.87600 & 1.31300 \\
\hline $\mathrm{H}$ & -4.81600 & 3.45100 & 2.81500 \\
\hline $\mathrm{H}$ & -4.95700 & 5.18500 & 2.42200 \\
\hline $\mathrm{H}$ & -3.74600 & 4.18100 & 1.58200 \\
\hline $\mathrm{H}$ & -3.47700 & -0.90000 & -0.91900 \\
\hline $\mathrm{H}$ & -5.27000 & -2.05900 & 0.18400 \\
\hline $\mathrm{H}$ & -5.81600 & -0.54600 & 2.03500 \\
\hline $\mathrm{H}$ & -4.20000 & -0.79100 & 2.74400 \\
\hline $\mathrm{H}$ & -4.86200 & -3.05400 & 2.85800 \\
\hline $\mathrm{H}$ & -1.29000 & -2.87100 & 2.26700 \\
\hline - & 0.91800 & -3.03300 & 1.18500 \\
\hline $\mathrm{H}$ & -1.02200 & -3.10900 & -2.65500 \\
\hline $\mathrm{H}$ & 1.41500 & -2.78800 & -2.55000 \\
\hline $\mathrm{H}$ & 2.65700 & -3.73300 & 0.08800 \\
\hline $\mathrm{H}$ & 3.78600 & -2.94700 & -2.66800 \\
\hline 1 & 4.30700 & -4.39300 & -1.79000 \\
\hline 1 & 5.27000 & -1.98200 & -1.51800 \\
\hline 1 & -5.09800 & -4.05800 & -2.22800 \\
\hline $\mathrm{H}$ & -3.66500 & -5.06900 & -1.90600 \\
\hline П & -4.55600 & -4.32600 & -0.54700 \\
\hline 1 & -2.60800 & 0.90500 & -1.73600 \\
\hline П & 1.78500 & 0.04800 & 0.37900 \\
\hline$\Gamma$ & -0.56300 & 1.21600 & -3.05700 \\
\hline$\Gamma$ & -0.49900 & 0.28600 & 3.87100 \\
\hline $\mathrm{H}$ & -1.76600 & 0.84400 & 2.74900 \\
\hline $\mathrm{H}$ & -0.16100 & 1.63700 & 2.75900 \\
\hline
\end{tabular}




\begin{tabular}{|c|c|c|c|}
\hline $\mathrm{H}$ & 1.82300 & 1.07000 & -3.30800 \\
\hline $\mathrm{H}$ & 3.17300 & -0.78100 & -1.40400 \\
\hline $\mathrm{H}$ & 5.74400 & 3.35000 & 1.78600 \\
\hline $\mathrm{H}$ & 4.48100 & -0.54500 & 0.39200 \\
\hline $\mathrm{H}$ & 4.31300 & 0.96400 & -3.63000 \\
\hline $\mathrm{H}$ & 3.71600 & -0.70000 & -3.79300 \\
\hline $\mathrm{H}$ & 5.96700 & 0.11900 & -2.31100 \\
\hline $\mathrm{H}$ & 2.97200 & 5.13400 & -1.00500 \\
\hline $\mathrm{H}$ & 4.39700 & 5.35800 & -2.04000 \\
\hline $\mathrm{H}$ & 4.12200 & 6.46000 & -0.65500 \\
\hline $\mathrm{H}$ & 6.26500 & 1.45000 & 3.11500 \\
\hline $\mathrm{H}$ & 5.64100 & -1.32300 & 1.94800 \\
\hline $\mathrm{H}$ & 5.94600 & -1.66400 & 4.48000 \\
\hline $\mathrm{H}$ & 7.02800 & -0.26400 & 4.49700 \\
\hline $\mathrm{H}$ & 8.32400 & -1.57200 & 2.98700 \\
\hline \multicolumn{4}{|l|}{$\alpha-\beta$} \\
\hline O & 8.08300 & -1.78900 & -1.62100 \\
\hline$C$ & 6.94700 & -1.22500 & -1.14700 \\
\hline$C$ & 4.60100 & -0.07600 & -0.17400 \\
\hline$C$ & 6.03000 & -1.99600 & -0.41400 \\
\hline$C$ & 6.67900 & 0.11000 & -1.39000 \\
\hline$C$ & 5.50400 & 0.68400 & -0.90600 \\
\hline$C$ & 4.86500 & -1.42600 & 0.07300 \\
\hline $\mathrm{O}$ & 6.40300 & -3.29900 & -0.25400 \\
\hline$C$ & 5.52700 & -4.14600 & 0.46500 \\
\hline$C$ & 3.35100 & 0.57400 & 0.37400 \\
\hline $\mathrm{C}$ & 3.65100 & 1.47200 & 1.57500 \\
\hline 0 & 2.45000 & 2.06700 & 2.08900 \\
\hline$C$ & 4.24600 & 0.72800 & 2.75900 \\
\hline 0 & 4.42900 & 1.59900 & 3.85400 \\
\hline $\mathrm{O}$ & 2.39100 & -0.37600 & 0.82000 \\
\hline$C$ & 1.49700 & 2.49800 & 1.20000 \\
\hline
\end{tabular}




\begin{tabular}{|c|c|c|c|}
\hline C & -0.51000 & 3.34100 & -0.57000 \\
\hline C & 1.78400 & 3.44600 & 0.21300 \\
\hline C & 0.21700 & 1.96200 & 1.28400 \\
\hline C & -0.77700 & 2.37700 & 0.40900 \\
\hline C & 0.78900 & 3.84800 & -0.66700 \\
\hline 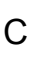 & -1.55200 & 3.86500 & -1.47000 \\
\hline$c$ & -2.85200 & 3.93700 & -1.18300 \\
\hline C & -3.88400 & 4.53400 & -2.09400 \\
\hline C & -4.93400 & 3.62900 & -2.37300 \\
\hline C & 3.05500 & 3.94300 & 0.06100 \\
\hline C & 3.47000 & 4.81100 & 1.11500 \\
\hline C & 1.44000 & -0.78700 & -0.06200 \\
\hline C & -0.69200 & -1.60800 & -1.73700 \\
\hline C & 1.58400 & -0.75200 & -1.45000 \\
\hline$c$ & 0.23600 & -1.26200 & 0.48900 \\
\hline$c$ & -0.80700 & -1.65500 & -0.32000 \\
\hline C & 0.54300 & -1.14900 & -2.26600 \\
\hline$c$ & 0.05900 & -1.27900 & 1.84700 \\
\hline C & 0.83800 & -2.25400 & 2.53000 \\
\hline C & -1.66500 & -2.00200 & -2.67900 \\
\hline C & -2.93500 & -2.45300 & -2.46800 \\
\hline C & -3.70000 & 0.34400 & -1.80600 \\
\hline 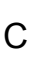 & -3.93100 & -0.78800 & -0.98100 \\
\hline 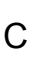 & -4.61900 & -2.92800 & 0.72600 \\
\hline c & -3.61100 & -2.13000 & -1.31300 \\
\hline c & -4.53700 & -0.57400 & 0.28200 \\
\hline C & -4.89400 & -1.61400 & 1.12700 \\
\hline C & -3.98400 & -3.18300 & -0.48300 \\
\hline c & -3.72800 & -4.47600 & -0.84300 \\
\hline 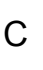 & -3.36200 & 0.41300 & -3.26000 \\
\hline 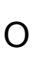 & -4.20900 & 1.36200 & -3.92000 \\
\hline & -2.37400 & -4.87800 & -0.66300 \\
\hline
\end{tabular}




\begin{tabular}{|c|c|c|c|}
\hline 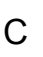 & -5.56000 & -1.38900 & 2.42200 \\
\hline ; & -6.20700 & -0.28400 & 2.79700 \\
\hline C & -6.85600 & -0.12600 & 4.13800 \\
\hline O & -8.23500 & 0.19000 & 4.02600 \\
\hline $\mathrm{H}$ & 8.09200 & -2.71700 & -1.35600 \\
\hline $\mathrm{H}$ & 7.39400 & 0.68500 & -1.96500 \\
\hline $\mathrm{H}$ & 5.29000 & 1.72800 & -1.10900 \\
\hline $\mathrm{H}$ & 4.14700 & -2.00900 & 0.63600 \\
\hline $\mathrm{H}$ & 5.39700 & -3.78900 & 1.49000 \\
\hline $\mathrm{H}$ & 5.99300 & -5.12800 & 0.47600 \\
\hline $\mathrm{H}$ & 4.55300 & -4.20600 & -0.03000 \\
\hline $\mathrm{H}$ & 2.90000 & 1.19600 & -0.40600 \\
\hline $\mathrm{H}$ & 4.34400 & 2.25500 & 1.25000 \\
\hline $\mathrm{H}$ & 5.22900 & 0.33900 & 2.49000 \\
\hline$H$ & 3.58900 & -0.10900 & 3.02200 \\
\hline$H$ & 3.57100 & 1.99400 & 4.04300 \\
\hline$H$ & 0.03600 & 1.18400 & 2.01700 \\
\hline $\mathrm{H}$ & -1.76000 & 1.92200 & 0.46700 \\
\hline $\mathrm{H}$ & 1.04300 & 4.58400 & -1.42200 \\
\hline $\mathrm{H}$ & -1.20300 & 4.27600 & -2.41600 \\
\hline $\mathrm{H}$ & -3.22300 & 3.57100 & -0.22700 \\
\hline $\mathrm{H}$ & -3.40600 & 4.89100 & -3.01500 \\
\hline $\mathrm{H}$ & -4.35000 & 5.39500 & -1.60700 \\
\hline$\pi$ & -4.59000 & 2.93300 & -2.95100 \\
\hline 11 & 4.47200 & 5.15000 & 0.85800 \\
\hline$\Pi$ & 2.79600 & 5.66900 & 1.18200 \\
\hline 11 & 3.49200 & 4.28400 & 2.07300 \\
\hline$\Pi$ & 2.51600 & -0.41800 & -1.89100 \\
\hline$\Pi$ & -1.70500 & -1.98900 & 0.18100 \\
\hline$\Pi$ & 0.67200 & -1.11400 & -3.34100 \\
\hline$\Pi$ & 0.58300 & -2.16900 & 3.58400 \\
\hline $\mathrm{H}$ & 1.90600 & -2.06700 & 2.39400 \\
\hline
\end{tabular}




\begin{tabular}{|c|c|c|c|}
\hline $\mathrm{H}$ & 0.58500 & -3.25700 & 2.17100 \\
\hline $\mathrm{H}$ & -1.41700 & -2.03800 & -3.73100 \\
\hline $\mathrm{H}$ & -3.91500 & 1.31100 & -1.36100 \\
\hline $\mathrm{H}$ & -4.89100 & -3.77300 & 1.35000 \\
\hline $\mathrm{H}$ & -4.73600 & 0.45200 & 0.57200 \\
\hline $\mathrm{H}$ & -3.44300 & -0.55500 & -3.75500 \\
\hline $\mathrm{H}$ & -2.34400 & 0.78900 & -3.40400 \\
\hline $\mathrm{H}$ & -5.09500 & 0.99000 & -3.96900 \\
\hline $\mathrm{H}$ & -2.07400 & -4.74700 & 0.38200 \\
\hline $\mathrm{H}$ & -1.70000 & -4.31200 & -1.31000 \\
\hline $\mathrm{H}$ & -2.33000 & -5.93300 & -0.92600 \\
\hline $\mathrm{H}$ & -5.51700 & -2.22500 & 3.11900 \\
\hline $\mathrm{H}$ & -6.31600 & 0.55900 & 2.11900 \\
\hline $\mathrm{H}$ & -6.41200 & 0.71200 & 4.68000 \\
\hline $\mathrm{H}$ & -6.70300 & -1.03200 & 4.73700 \\
\hline $\mathrm{H}$ & -8.65500 & -0.49100 & 3.49500 \\
\hline \multicolumn{4}{|l|}{$0-4$} \\
\hline $\mathrm{O}$ & -8.36500 & 1.45600 & -1.57800 \\
\hline$C$ & -7.18400 & 0.93200 & -1.17400 \\
\hline C & -4.74700 & -0.13500 & -0.34200 \\
\hline$C$ & -6.36800 & 1.64100 & -0.27700 \\
\hline$C$ & -6.77000 & -0.30000 & -1.65000 \\
\hline$C$ & -5.55000 & -0.83100 & -1.23700 \\
\hline$C$ & -5.15700 & 1.11000 & 0.13900 \\
\hline $\mathrm{O}$ & -6.88100 & 2.84500 & 0.10700 \\
\hline$C$ & -6.11500 & 3.62500 & 1.00500 \\
\hline$C$ & -3.44500 & -0.74800 & 0.12000 \\
\hline$C$ & -3.66400 & -1.87600 & 1.13000 \\
\hline $\mathrm{O}$ & -2.42000 & -2.44900 & 1.56900 \\
\hline$C$ & -4.34700 & -1.42000 & 2.41000 \\
\hline $\mathrm{O}$ & -4.46100 & -2.48800 & 3.32400 \\
\hline 0 & -2.59200 & 0.20300 & 0.74700 \\
\hline
\end{tabular}




\begin{tabular}{|c|c|c|c|}
\hline C & -1.35800 & -2.49800 & 0.70200 \\
\hline ; & 0.84200 & -2.50000 & -1.03800 \\
\hline C & -1.45600 & -3.09800 & -0.55800 \\
\hline C & -0.15600 & -1.91700 & 1.09000 \\
\hline C & 0.93500 & -1.92100 & 0.23300 \\
\hline C & -0.37200 & -3.07200 & -1.42400 \\
\hline C & 1.99500 & -2.53500 & -1.95800 \\
\hline C & 3.26400 & -2.66200 & -1.57000 \\
\hline C & 4.44800 & -2.70900 & -2.49300 \\
\hline O & 5.43200 & -1.75600 & -2.13000 \\
\hline O & -2.64400 & -3.64600 & -0.97900 \\
\hline C & -2.99500 & -4.85200 & -0.30100 \\
\hline C & -1.60300 & 0.76600 & 0.00500 \\
\hline$C$ & 0.59700 & 1.82600 & -1.35300 \\
\hline$c$ & -1.60700 & 0.83200 & -1.38400 \\
\hline$C$ & -0.51300 & 1.29000 & 0.72100 \\
\hline$C$ & 0.57200 & 1.81700 & 0.04500 \\
\hline$C$ & -0.50800 & 1.36100 & -2.05500 \\
\hline $\mathrm{O}$ & -0.47300 & 1.20400 & 2.08500 \\
\hline C & -1.40100 & 2.04300 & 2.76300 \\
\hline C & 1.81500 & 2.33300 & -2.12600 \\
\hline $\mathrm{O}$ & 1.95700 & 3.64400 & -1.73300 \\
\hline C & 3.08200 & 1.48100 & -1.90900 \\
\hline C & 3.67500 & 1.59100 & -0.52600 \\
\hline C & 4.79700 & 1.78200 & 2.04600 \\
\hline C & 3.88700 & 2.80100 & 0.10300 \\
\hline C & 4.09500 & 0.44900 & 0.15400 \\
\hline C & 4.67100 & 0.53800 & 1.43100 \\
\hline C & 4.40200 & 2.94600 & 1.37400 \\
\hline $\mathrm{O}$ & 4.54600 & 4.13400 & 2.00200 \\
\hline C & 4.13900 & 1.86000 & -2.95300 \\
\hline O & 5.28900 & 1.03700 & -2.85600 \\
\hline
\end{tabular}




\begin{tabular}{|c|c|c|c|}
\hline C & 4.09000 & 5.26400 & 1.27400 \\
\hline 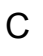 & 5.14000 & -0.67500 & 2.12500 \\
\hline C & 5.53400 & -1.79600 & 1.51500 \\
\hline C & 5.98000 & -3.01700 & 2.26000 \\
\hline $\mathrm{O}$ & 7.26500 & -3.45700 & 1.85000 \\
\hline $\mathrm{H}$ & -8.47800 & 2.31100 & -1.14500 \\
\hline $\mathrm{H}$ & -7.40900 & -0.82500 & -2.34800 \\
\hline $\mathrm{H}$ & -5.21900 & -1.79100 & -1.62200 \\
\hline $\mathrm{H}$ & -4.51700 & 1.64700 & 0.82600 \\
\hline $\mathrm{H}$ & -5.96800 & 3.09500 & 1.95100 \\
\hline $\mathrm{H}$ & -6.68500 & 4.53400 & 1.18100 \\
\hline $\mathrm{H}$ & -5.14400 & 3.87800 & 0.56900 \\
\hline $\mathrm{H}$ & -2.93500 & -1.17700 & -0.74400 \\
\hline $\mathrm{H}$ & -4.27100 & -2.64900 & 0.64800 \\
\hline$H$ & -5.35900 & -1.08200 & 2.18200 \\
\hline $\mathrm{H}$ & -3.77800 & -0.58700 & 2.83900 \\
\hline $\mathrm{H}$ & -3.57300 & -2.83400 & 3.46300 \\
\hline $\mathrm{H}$ & -0.11800 & -1.41000 & 2.04700 \\
\hline $\mathrm{H}$ & 1.85300 & -1.42900 & 0.53800 \\
\hline $\mathrm{H}$ & -0.48900 & -3.52700 & -2.40100 \\
\hline 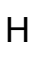 & 1.76700 & -2.49100 & -3.02300 \\
\hline 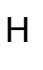 & 3.49300 & -2.76000 & -0.51000 \\
\hline 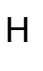 & 4.12300 & -2.57900 & -3.53200 \\
\hline$\Pi$ & 4.93200 & -3.68600 & -2.41600 \\
\hline$\pi$ & 5.21500 & -0.89200 & -2.51000 \\
\hline$\pi$ & -3.94600 & -5.17600 & -0.71800 \\
\hline 11 & -2.23400 & -5.61700 & -0.47800 \\
\hline$\Pi$ & -3.10000 & -4.68100 & 0.77400 \\
\hline$\Pi$ & -2.46000 & 0.47500 & -1.94600 \\
\hline$\Pi$ & 1.40200 & 2.19200 & 0.63100 \\
\hline $\mathrm{H}$ & -0.51700 & 1.39800 & -3.13800 \\
\hline $\mathrm{H}$ & -1.23300 & 1.89100 & 3.82700 \\
\hline
\end{tabular}




$\begin{array}{rrrr}\mathrm{H} & -2.42900 & 1.77200 & 2.51200 \\ \mathrm{H} & -1.21800 & 3.09200 & 2.50900 \\ \mathrm{H} & 1.54500 & 2.28900 & -3.19200 \\ \mathrm{H} & 2.80100 & 0.43400 & -2.08000 \\ \mathrm{H} & 5.21400 & 1.87600 & 3.04200 \\ \mathrm{H} & 3.96500 & -0.51800 & -0.31300 \\ \mathrm{H} & 4.40800 & 2.91800 & -2.84000 \\ \mathrm{H} & 3.74400 & 1.71000 & -3.96000 \\ \mathrm{H} & 5.73300 & 1.23500 & -2.02200 \\ \mathrm{H} & 3.02800 & 5.16600 & 1.03200 \\ \mathrm{H} & 4.65500 & 5.37800 & 0.34400 \\ \mathrm{H} & 4.25200 & 6.12500 & 1.91700 \\ \mathrm{H} & 5.17400 & -0.62400 & 3.21200 \\ \mathrm{H} & 5.57500 & -1.85300 & 0.42700 \\ \mathrm{H} & 5.30900 & -3.85500 & 2.05000 \\ \mathrm{H} & 5.95100 & -2.82900 & 3.34100 \\ \mathrm{H} & 7.86400 & -2.70700 & 1.88900\end{array}$


S.3. Spin density plots for the RSRS Stereoisomer

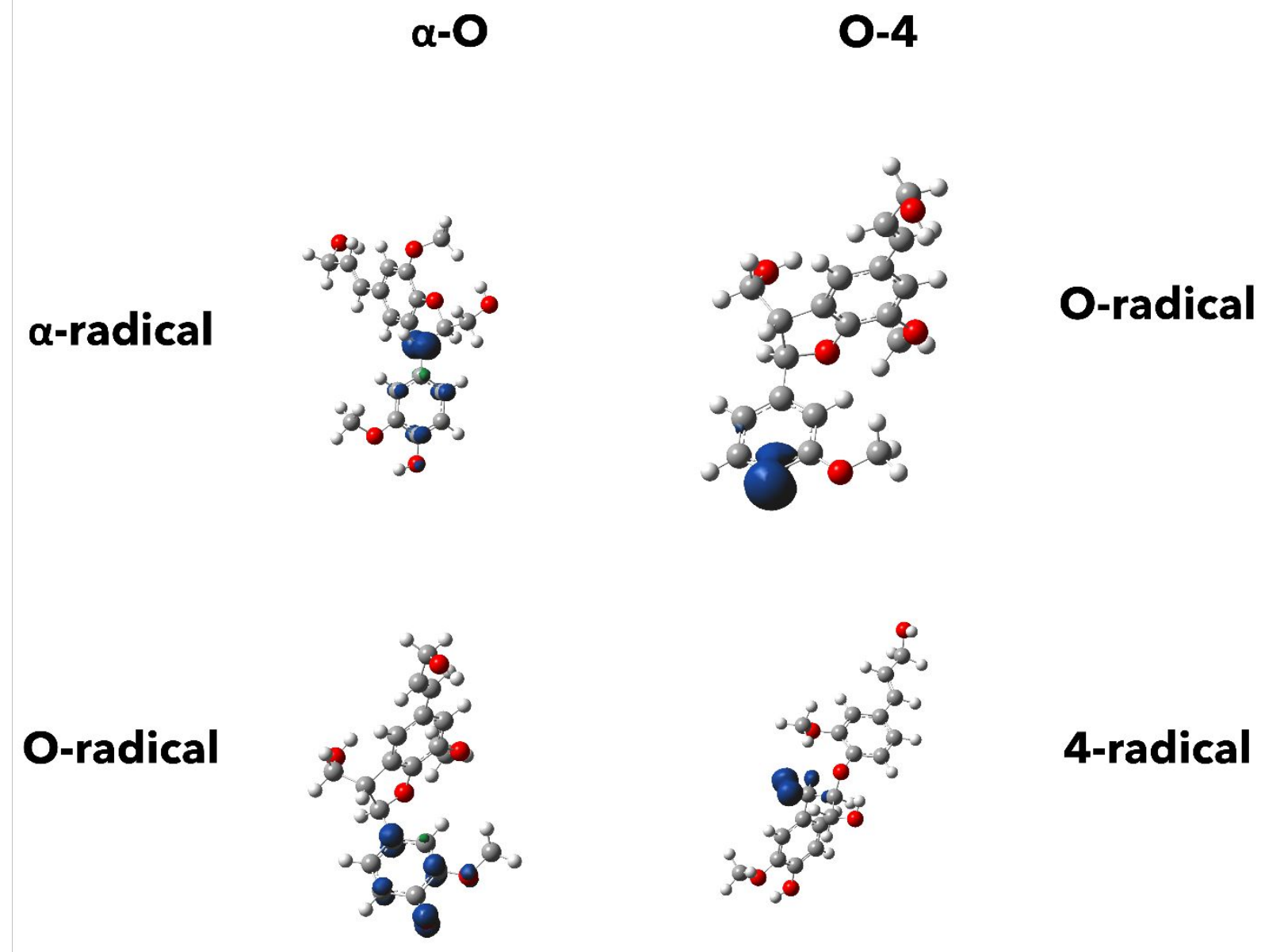

Figure S.3.1. Spin density plot for the $\alpha-\mathrm{O}-4$ bond scission products of the RSRS stereoisomer. 
1- $\alpha$
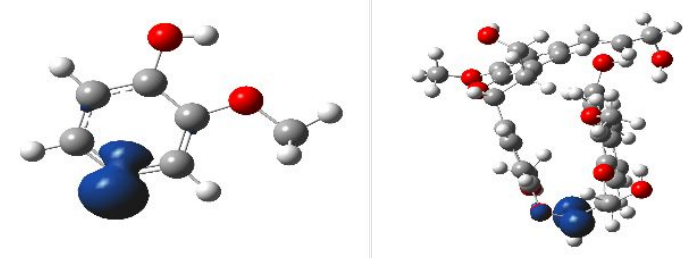

$\alpha-\beta$
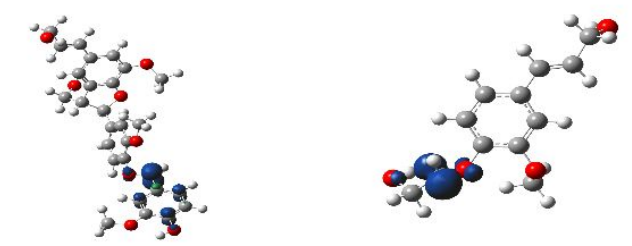

$\beta-0$
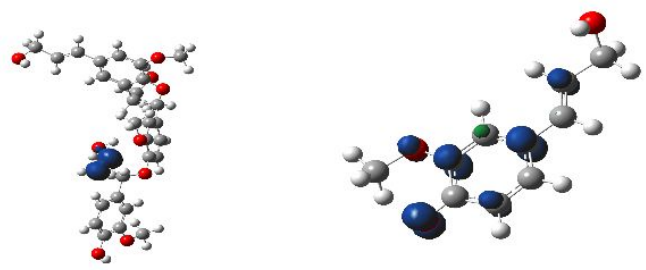

0-4
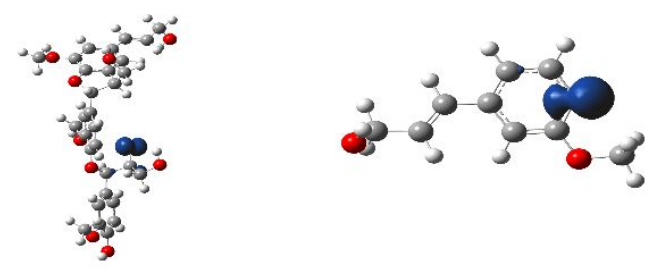

$\boldsymbol{\gamma} \boldsymbol{\beta}$

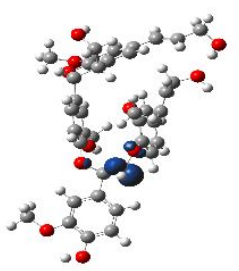

Y-O

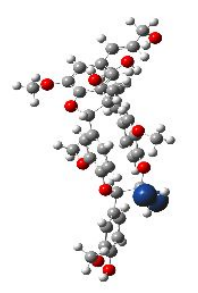

Figure S.3.2. Spin density plot for the $\beta-\mathrm{O}-4$ bond scission products of the RSRS stereoisomer. 
$1^{\text {st }}$-radical

a-Phenyl

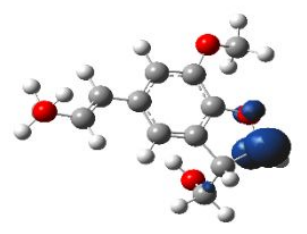

$\alpha-\beta$

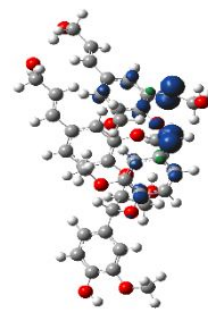

$\alpha-0$

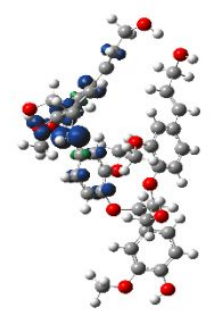

$\beta-5$

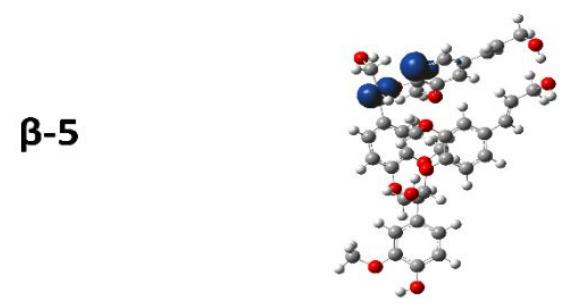

0-4 2nd-radical

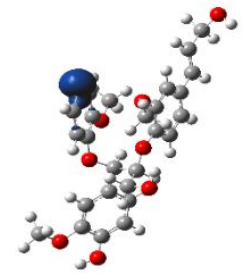

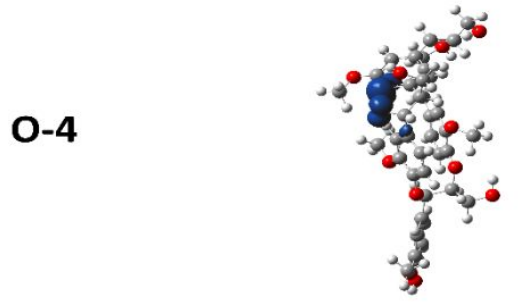

Figure S.3.3. Spin density plot for the $\beta-5$ bond scission products of the RSRS stereoisomer. 